\title{
Unspoken aspects of chain shuttling reactions: Patterning the molecular landscape of olefin multi-block copolymers
}

Mohammad Reza Saeb ${ }^{1}$, Yousef Mohammadi 2*, Tayebeh Sadat Kermaniyan ${ }^{3}$, Philippe Zinck ${ }^{4}$, Florian J. Stadler ${ }^{5 *}$

${ }^{1}$ Department of Resin and Additives, Institute for Color Science and Technology, P.O. Box 16765-654, Tehran, Iran.

${ }^{2}$ Petrochemical Research and Technology Company (NPC-rt), National Petrochemical Company (NPC), P.O. Box 14358-84711, Tehran, Iran.

${ }^{3}$ Department of Polymer Engineering and Color Technology, Amirkabir University of Technology, Tehran, Iran.

${ }^{4}$ University of Lille, ENSCL, UCCS, UMR CNRS 8181, Bât C7, Cité Scientifique, 59652 Villeneuve d'Ascq Cédex, France.

${ }^{5}$ College of Materials Science and Engineering, Shenzhen Key Laboratory of Polymer Science and Technology, Guangdong Research Center for Interfacial Engineering of Functional Materials, Nanshan District Key Lab for Biopolymers and Safety Evaluation, Shenzhen University, Shenzhen 518060, China.

To whom correspondence may be addressed:

1- Dr. Yousef Mohammadi, E-mail: mohammadi@npc-rt.ir, Tel.: +98-021-44787510.

2- Prof. Florian J. Stadler, E-mail: fjstadler@szu.edu.cn, Tel.: +86-0755-26538783, Telefax: +860755-26536239. 


\begin{abstract}
Molecular landscape of olefin block copolymers (OBCs) was patterned by hybridizing capabilities of Kinetic Monte Carlo (KMC) and Artificial Neural Network (ANN) stochastic modeling approaches to explore complexities with chain shuttling copolymerization of ethylene with $\alpha$ olefins. Theoretical data on chain microstructure were obtained by an in-house KMC simulator. The interdependence between microstructure and operating conditions was uncovered by ANN modeling. The average number of linkage points per OBC chain is monitored as a direct criterion reflecting the multi-block nature of OBCs. We also quantified hard and soft block length and ethylene sequence length of both blocks in terms of catalyst composition, ethylene to 1-octene ratio, and chain shuttling agent level, giving useful insights to be applied to developing tailored OBCs. The proposed hybrid stochastic modeling approach successfully predicts the conditions for producing $\mathrm{OBCs}$ with predesigned structure; i.e., block length, block number, and ethylene sequence length in hard and soft segments of OBC. As a unique feature of this work, we suggest operation condition for developing and identifying new families of OBCs with microstructures that were previously unexplored.
\end{abstract}

Keywords: Olefin multi-block copolymer; artificial neural network; chain shuttling reaction; tailored copolymer; Molecular pattern 


\section{Introduction}

The advent of olefin block copolymers (OBCs) with alternating semicrystalline hard and soft segments opened a new window to polymerization reaction engineers, while confronting them with continuous upward challenges in elucidating complexities associated with chain shuttling reactions $[1,2]$. This seminal work, represented in Scheme 1, has inspired many chemists, extending the concept to various new and original multiblock microstructures and architectures, and developing new catalytic combinations [3-9].

Cat 1: Hafnium pyridylamide catalyst (High comonomer incorporation ability)

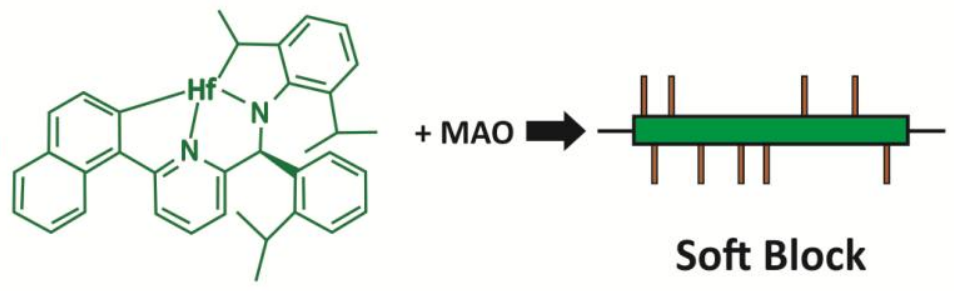

1
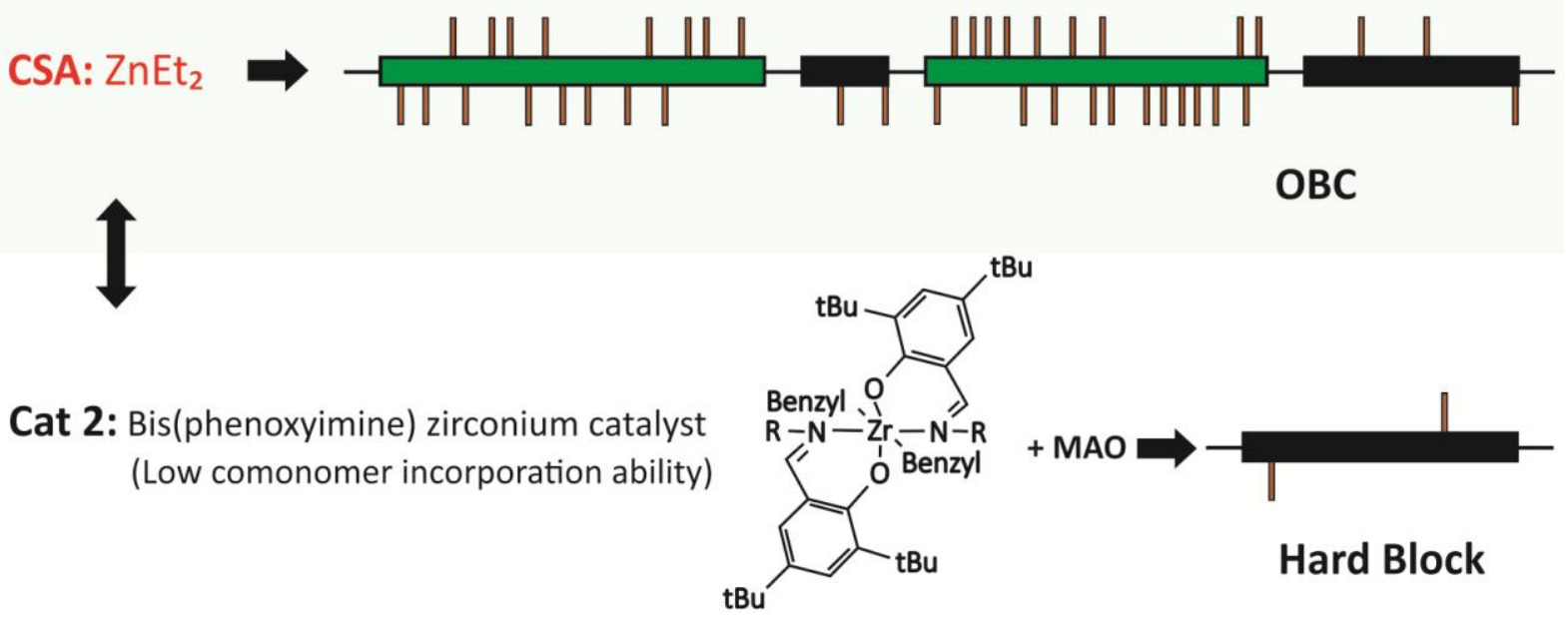

Scheme 1. Ethylene / 1-octene chain shuttling polymerization leading to OBC. CSA is the chain shuttling agent and MAO methylaluminoxane. $\mathrm{R}=2$-methylcyclohexyl.

The quasi-living nature of chain shuttling copolymerization accounts for competitive formation of both dormant and living copolymer chains in a one-step polymerization. The presence of a chain shuttling agent (CSA) in a dual-catalyst system causes the exchange of active 
centers between copolymer chains proceeding self- and cross-shuttling reactions. As a result, depending on catalyst composition, monomer ratio, and CSA concentration, a large variety of copolymers with different blockiness signature are possible [10]. Nevertheless, it would be naive to think that control/identification of chain shuttling reaction is straightforward, as even when finding a proper CSA and catalyst combination, which itself is a cumbersome task, deactivation of catalyst moieties may cause a significant deviation from the desired OBC microstructure [11].

We recently published three consecutive papers on Kinetic Monte Carlo (KMC) simulation of chain shuttling copolymerization of ethylene with 1-octene [12-14]. The developed in-house computer algorithm revealed capability of synthesizing, characterizing, and screening a huge number of copolymer chains, both instantaneously and cumulatively. As a result, different characteristics of $\mathrm{OBC}$ chains (hard block weight fraction, number of hard and soft blocks per chain, average number of linkage points per chain (LP), chemical composition distribution (CCD), and molecular weight distribution) and blocks (average degree of polymerization of hard $\left(\overline{\mathrm{DP}}_{\mathrm{n}}^{\mathrm{HARD}}\right)$ and soft $\left(\overline{\mathrm{DP}}_{\mathrm{n}}^{\mathrm{SOFT}}\right)$ blocks, number of $\alpha$-olefin units per block, CCD of soft and hard blocks, distribution of degree of polymerization of hard and soft blocks, average number of ethylenic sequences in the blocks, and ethylene sequence length of hard ( $\overline{\mathrm{ESL}} \mathrm{HARD})$ and soft $\left(\overline{\mathrm{ESL}}{ }^{\mathrm{SOFT}}\right.$ ) segments) have been enumerated by the computer-aided KMC algorithm.

The multitude and amount of data of KMC simulator is too large and complex to be analyzed manually. In addition, $\mathrm{KMC}$ is time-consuming and optimization of polymerization for desired OBC proves difficult. The runtime of KMC code was found to be, on average, 10 hours, which requires more than two months for simulation of 200 OBC types in this paper [12]. Moreover, KMC cannot identify and generalize non-linear dynamic behavior of chain shuttling reaction, e.g. for controlling the average number of blocks per chain by manipulating operating conditions. Thus, an advanced cognitive tool with sufficient statistical power for demonstrating the complex association between operating conditions and macromolecular features of OBC needs to assist KMC simulator in simulation. This diminishes calculation time from hundreds of years (assuming that all possible recipes would be calculated) to just less than two hours. 
For identifying the behavior of a given system, one can use classical or stochastic modeling approaches $[15,16]$. Classical mathematical rules express a process in terms of a set of formal mathematical functions, while stochastic modeling approaches are based on non-linear mappings $[17,18]$. If such capabilities are merged in an efficient way, the resulting black-box learns the behavior of system and finds relationships between input and output variables. Artificial neural network (ANN) is an intelligent black-box modeling approach with an acceptable computational time and accuracy, which can be applied to model complex processes. ANN has occasionally been considered for modeling polymerization process, where mechanistic description of the interrelationship between operating conditions and polymer properties was unknown/complex, but still unable to understand molecular features [19-27]. The analysis of OBCs is still in its infancy due to the lack of a suitable number of materials having been produced and properly analyzed. In the current paper, we apply ANN modeling to visualize the latent fingerprint of chain shuttling reactions.

Production of OBCs needs a multifaceted probability analysis, as vast structural complexities are brought for each structure in view of block type and block-block juncture [28]. KMC simulator was put into run to simulate 175 scenarios identical to 175 OBCs. These scenarios were fed into the ANN to find the connections between input and output variables and to explicitly illustrate new families of OBC with the aid of discovered microstructural patterns. Here, the question that at what operating conditions OBCs with tailored microstructures can be produced is answered, and new grades of OBCs with predesigned LP, $\overline{\mathrm{DP}}_{\mathrm{n}}^{\mathrm{HARD}}, \overline{\mathrm{DP}}_{\mathrm{n}}^{\mathrm{SOFT}}$, $\overline{\text { ESL }}$ HARD, and $\overline{\text { ESL }}$ SOFT are defined, quantified, and patterned, by which bivariate plots with distinguished macromolecular landscapes are specified for tailoring OBC.

\section{Model development}

A two-step modeling procedure has been applied in this work. In the first step, KMC simulator was used to synthesize macromolecules with different architectural features [12-14]. In a nutshell, this KMC simulator assumes a reactor containing $10^{11}$ molecules. Two types of catalyst are inserted, which differ in their comonomer uptake rate. Catalyst 1 (Hf based, see 
Scheme 1) is 6 times more likely to incorporate 1-octene comonomer than Catalyst 2 (Zr based, see Scheme 1). Furthermore, Catalyst 1 has a 10 times higher homopropagation rate constant and a 100 times higher transfer to hydrogen rate constant than Catalyst 2. A CSA transfers active chains from one active center to another, which can be of the same or different type as the catalyst from which the CSA abstracted it. These constants are in accordance with the seminal work of Arriola et al. [1] and are summarized in Table 1. The simulation is run for simulated $600 \mathrm{~s}$ (taking $\approx 10 \mathrm{~h}$ in reality) assuming a batch type CSTR. Then the outcome is analyzed with respect to $\mathrm{LP}, \overline{\mathrm{DP}}_{\mathrm{n}}^{\mathrm{HARD}}, \overline{\mathrm{DP}}_{\mathrm{n}}^{\mathrm{SOFT}}, \overline{\mathrm{ESL}} \mathrm{HARD}$, and $\overline{\mathrm{ESL}} \mathrm{SOFT}$.

For this article, the following specified chain shuttling agent levels (log CSA Level: $-3,-2,-1,0$, $+1,+2,+3$; the CSA Level represents the quantity of CSA used in this work divided by the its quantity used in ref. 1, Table 1), catalyst composition (CC, which represents the molar ratio Catalyst $1 /$ Catalyst $2: 0.05,0.25,0.50,0.75,0.95)$, and monomer ratio (MR, which represents the molar ratio ethylene/1-octene : 0.05 (lowest octene content), 0.25, 0.50, 0.75, 0.95 (highest octene content) were used. Accordingly, 175 OBCs with various microstructural and architectural features were created in the simulation volume. As mentioned before, being meticulous when taking such relatively large search space will empower ANN in finding more scenarios with a higher degree of accuracy and precision. In the second step, we implemented ANN approach to uncover the interdependence between operating conditions and architectural features of synthesized macromolecules. Details on stochastic KMC simulation of chain shuttling copolymerization of ethylene with 1-octene can be found in our previous papers [12-14]. Some typical OBCs with detailed characteristics among 175 runs produced using in-house KMC simulator are summarized in Table 2.

Table 1. Parameters applied in KMC simulation.

\begin{tabular}{|c|c|c|}
\hline Kinetic Parameter & Catalyst 1 & Catalyst 2 \\
\hline Homopropagation rate constant ethylene $\left(\mathrm{L}^{\mathrm{mol}}{ }^{-1} \cdot \mathrm{s}^{-1}\right)$ & 100000 & 10000 \\
\hline Homopropagation rate constant 1-octene $\left(\mathrm{L} . \mathrm{mol}^{-1} \cdot \mathrm{s}^{-1}\right)$ & 1000 & 1000 \\
\hline Shuttling to CSA rate constant $\left(\right.$ L.mol $\left.{ }^{-1} \cdot \mathrm{s}^{-1}\right)$ & 1000000 & 1000000 \\
\hline Transfer to hydrogen rate constant $\left(\right.$ L.mol $\left.^{-1} \cdot \mathrm{s}^{-1}\right)$ & 5000 & 50 \\
\hline
\end{tabular}




\begin{tabular}{lll} 
Reactivity ratio of ethylene & 5 & 100 \\
Reactivity ratio of 1-octene & 0.3 & 0.05 \\
\hline Initial concentration of ethylene & 2.63 & $\mathrm{~mol} \mathrm{~L}^{-1}$ \\
Solvent initial mass & 1000 & $\mathrm{~g}$ \\
Ethylene initial mass & 200 & $\mathrm{~g}$ \\
1-octene initial mass & Variable based on MR value & $\mathrm{g}$ \\
Hydrogen initial mass & 0.072 & $\mathrm{~g}$ \\
CSA initial mass & 0.27 & $\mathrm{~g}$ \\
Catalyst metal initial mass & $1.5 \times 10^{-4}$ & $\mathrm{~g}$ \\
\end{tabular}

The scenario "Arriola et al." in Table 2 corresponds to the experimental run due to Arriola et al. [1]. This scenario was included to state which of the possible OBC-types were previously synthesized. The microstructural features of this special grade are obtained by our KMC simulator (Table 2). These values, which could not be principally expected from available experimental characterization methods, decoded the molecular signature of so far OBCs commercialized by DOW chemicals.

Table 2. Some typical OBCs produced by KMC simulator at specified operating conditions together with their molecular characteristics.

\begin{tabular}{l|lll|lllll}
\hline \multicolumn{3}{c}{ Inputs/Operating conditions } & \multicolumn{2}{l}{ Outputs/Microstructural features } \\
\hline $\begin{array}{l}\text { Scenario } \\
\text { No. }\end{array}$ & X1: MR & X2: CC & $\begin{array}{l}\text { X3: } \\
\log (\text { CSA Level) }\end{array}$ & Y1: $\overline{\text { DP }}$ SOFT & $\overline{\text { Y2: DP }}$ HARD & Y3: $\overline{\text { ESL }}$ S0FT & $\overline{\text { Y4: ESL HARD }}$ & Y5: LP \\
\hline 45 & 0.25 & 0.25 & -1 & 332.98 & 1323.07 & 2.82 & 36.33 & 2.06 \\
46 & 0.25 & 0.25 & 0 & 59.71 & 136.86 & 2.82 & 29.49 & 16.44 \\
47 & 0.25 & 0.25 & +1 & 7.90 & 14.97 & 2.84 & 10.70 & 43.66 \\
59 & 0.25 & 0.75 & -1 & 591.81 & 450.23 & 2.98 & 37.05 & 0.84 \\
60 & 0.25 & 0.75 & 0 & 158.59 & 46.54 & 2.99 & 21.66 & 7.32 \\
61 & 0.25 & 0.75 & +1 & 20.63 & 5.88 & 3.05 & 4.93 & 26.04 \\
115 & 0.75 & 0.25 & -1 & 1021.90 & 1270.87 & 21.39 & 306.29 & 2.05 \\
116 & 0.75 & 0.25 & 0 & 185.77 & 131.27 & 20.96 & 99.47 & 16.45 \\
117 & 0.75 & 0.25 & +1 & 26.21 & 14.12 & 16.95 & 13.66 & 43.69 \\
129 & 0.75 & 0.75 & -1 & 2173.82 & 432.77 & 32.43 & 245.63 & 0.85 \\
130 & 0.75 & 0.75 & 0 & 585.04 & 44.54 & 32.21 & 41.31 & 7.32 \\
131 & 0.75 & 0.75 & +1 & 79.21 & 5.41 & 29.58 & 5.31 & 26.07
\end{tabular}


Although not as detailed as the data sets we explored here, we learnt from our previous studies that LP is a direct measure of the blockiness. LP is essentially the number of blocks, but one should be aware that also two or more identical block types (hard or soft blocks) might be attached to each other directly, which, however, happens with the same likeliness for all cases with the same CC. In addition, experts know that $\overline{\mathrm{DP}}_{\mathrm{n}}^{\mathrm{HARD}}, \overline{\mathrm{DP}}_{\mathrm{n}}^{\mathrm{SOFT}}, \overline{\mathrm{ESL}}^{\mathrm{HARD}}$, and $\overline{\mathrm{ESL}}$ SOFT provide useful information about batch-to-batch transitions in blockiness of copolymers. Accordingly, five separate ANNs, corresponding to aforementioned target characteristics, are constructed, trained, and tested to capture complex nature of chain shuttling reaction in terms of CC, MR, and CSA level operating parameters. Each ANN was responsible for proficient prediction of one molecular feature of OBCs produced by KMC simulator. ANN takes the recipes and microstructural features produced by KMC simulator as input and output parameters, respectively. Then, it determines the microstructural features for thousands of new recipes, by which a large diversity of OBCs can be produced. The below procedure has been followed to model microstructural changes in terms of operating conditions.

Basically, modeling by ANN contains training and test procedures, through which weights and biases are manipulated to minimize errors in prediction of targets. In this manner, the constructed model learns the behavior of the polymerization process and reciprocally controls and tunes the model outputs comparing generated data with values obtained by KMC simulator in the first step of modeling until a minimum error is reached. Upon completion of the training, the educated ANN enables prediction of remainder of data sets in a similar fashion. The training step teaches the ANNs the complex behavior of chain shuttling reaction in terms of CC, MR, and CSA level. After completion of the training process, the ANNs gained the capability to predict the outputs based on any input data similar to the pattern they learned. For further information on ANN modeling, the reader is referred to section S1 in Supporting Information. 


\section{Results and discussion}

\subsection{ANN model verification}

To uncover the interdependence between operating conditions and microstructural features, it was essential first to evaluate the truthfulness of ANNs by comparing model predictions with the corresponding values of targets gained from KMC simulator at identical operating conditions. Figure 1 shows the variation of MSEs of the best chromosome in the training phase of the network, confirming that the behavior of the targets is predicted by the ANN models successfully. A further confirmation of model accuracy can be found looking at Figure 2, where prediction results are very close to the ideal $y=x$ line. The observed trends confirm that the developed ANNs can satisfactorily predict macromolecular patterns of OBCs with a quite small error. The statistical values in Table $\mathbf{3}$ indicate that the mean-squared error (MSE defined in S3 in Supporting Information) values of the optimized networks corresponding to training and test phases are reasonably low. Another beneficial feature of ANN modeling is the ability to identify and report the maximum errors in training and test phases, which are satisfactorily low. Moreover, coefficient of determination $\left(\mathrm{R}^{2}\right)$ corresponding to all studied responses was close to unity. Thus, the accuracy of the developed ANN in predicting the entire variable space, especially those cases that were not directly fed into the network during the training phase, has been verified. 


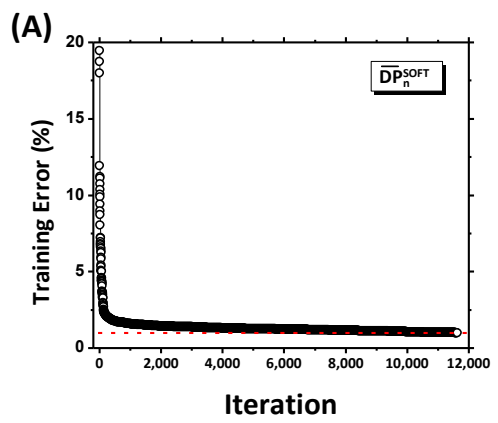

(B)
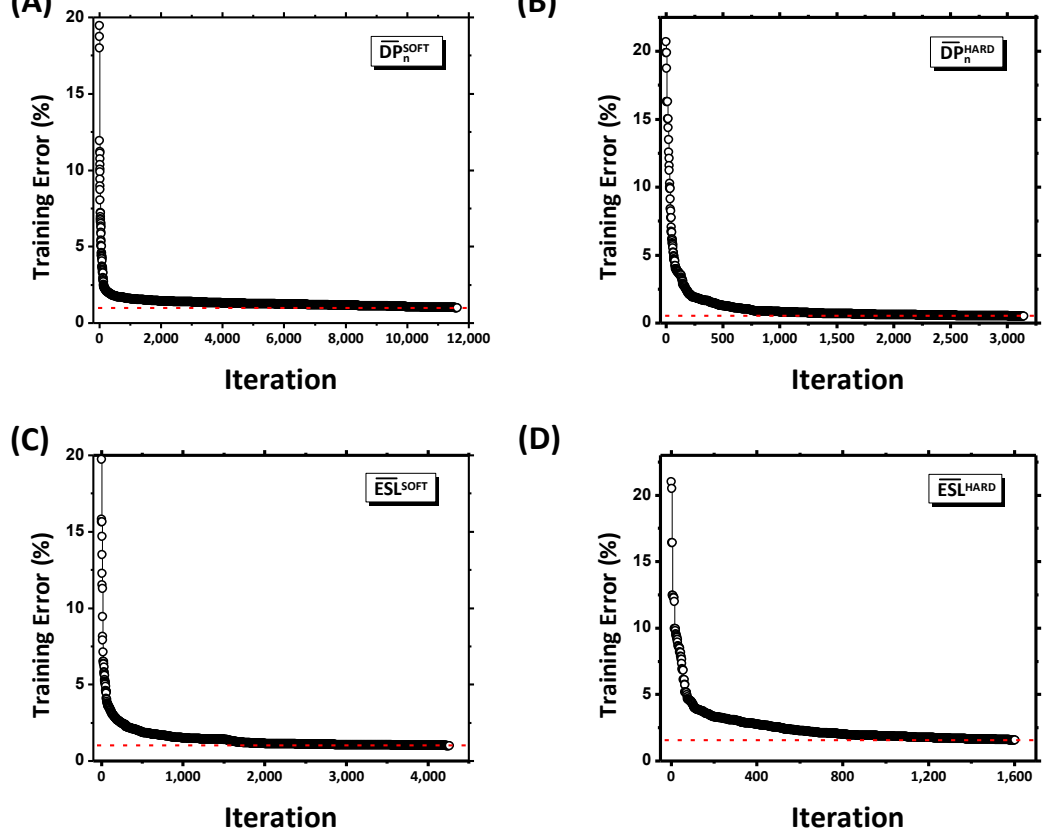

(D)
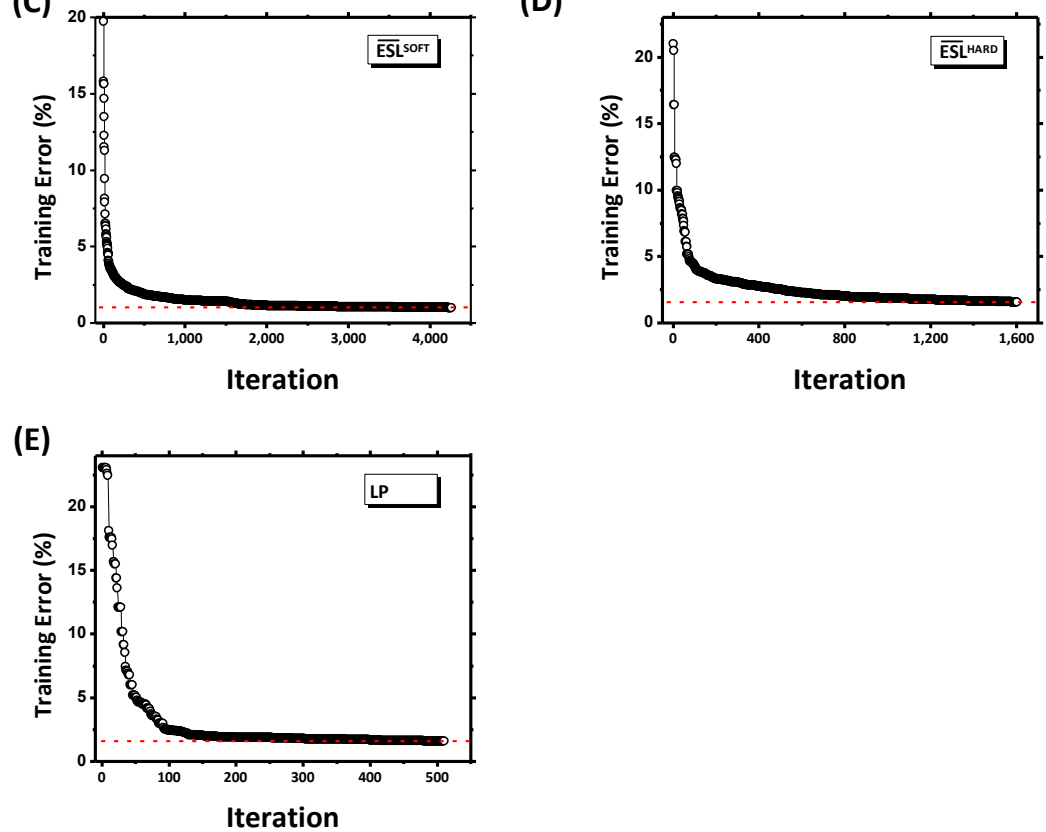

Figure 1. Iteration-dependence error in training phase of ANN modeling to reach an error lower than $1 \%$ in case of $Y_{1}-Y_{3}$ and $1.58 \%$ in case of $Y_{4}$ and $Y_{5}$ for (A) $\overline{D P}_{n}^{\text {SOFT }}$, (B) $\overline{D P}_{n}^{\text {HARD }}$, (C) $\overline{\text { ESL }}^{\text {HARD }}$, (D) $\overline{\text { ESL }}$ SOFT , and (E) LP. 


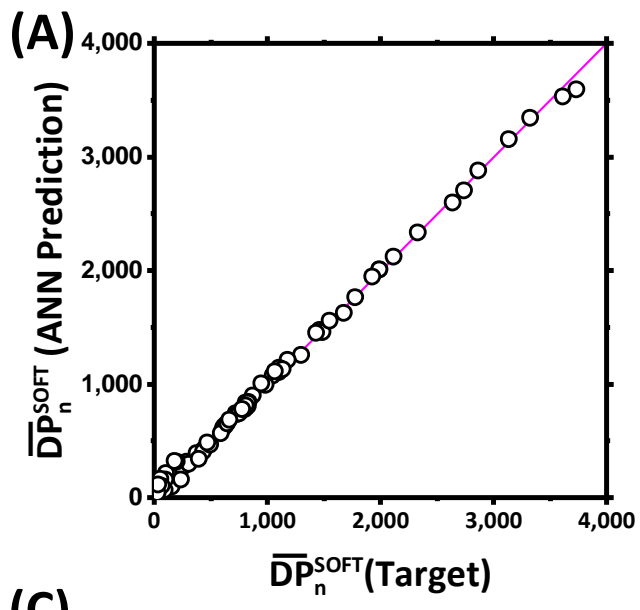

(C)

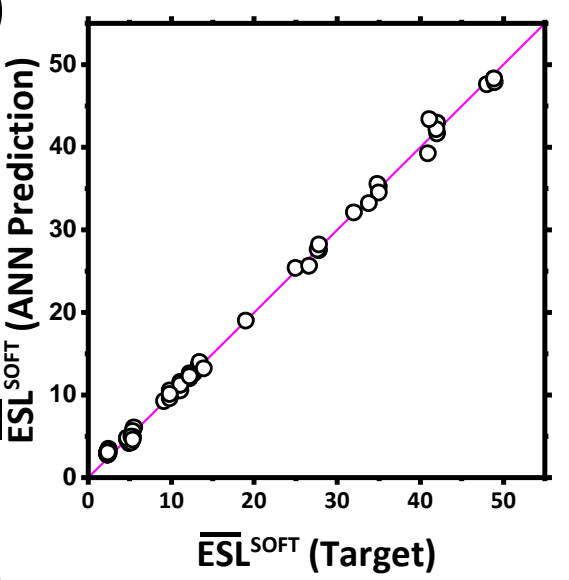

(E)

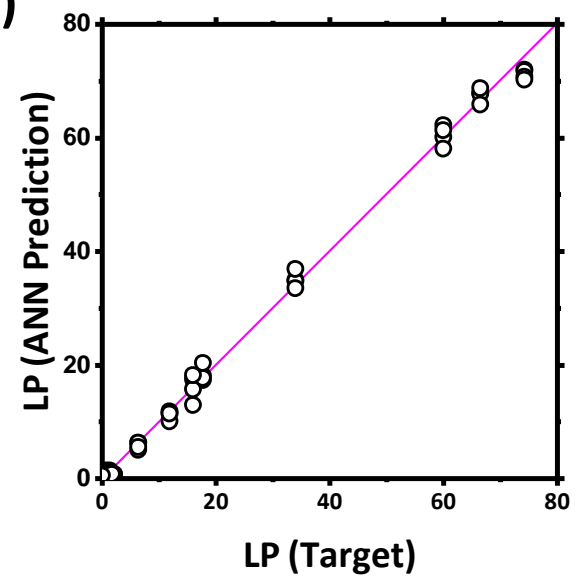

(B)

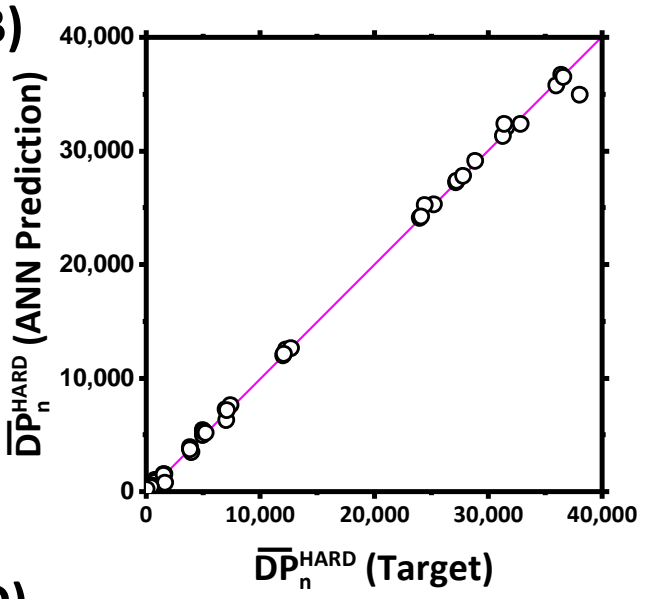

(D)

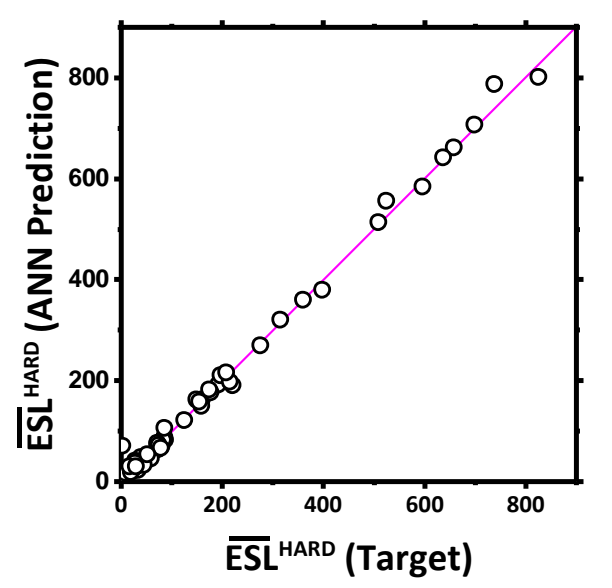

Figure 2. Correlation between the ANNs' predictions and equivalent data obtained by KMC simulator (A) $\overline{\mathrm{DP}}_{\mathrm{n}}^{\mathrm{SOFT}}$, (B) $\overline{\mathrm{DP}}_{\mathrm{n}}^{\mathrm{HARD}}$, (C) $\overline{\mathrm{ESL}}^{\text {HARD }}$, (D) $\overline{\mathrm{ESL}}$ SOFT , and (E) LP. 
Table 3. Results of statistical analyses related to artificial neural networks training and test procedures.

\begin{tabular}{llllll}
\hline & Y1 $\left(\overline{\mathbf{D P}}_{\mathbf{n}}^{\text {SOFT }}\right)$ & Y2 $\left(\overline{\mathbf{D P}}_{\mathbf{n}}^{\text {HARD }}\right)$ & Y3 $\left(\overline{\mathbf{E S L}}{ }^{\text {SOFT }}\right)$ & Y4 $\left(\overline{\mathbf{E S L}}{ }^{\text {HARD }}\right)$ & Y5 (LP) \\
\hline Training MSE & 0.00039 & 0.00039 & 0.00039 & 0.00099 & 0.00099 \\
Test MSE & 0.00109 & 0.00083 & 0.00125 & 0.00129 & 0.00137 \\
Training Error (\%) & 0.99987 & 0.99991 & 0.99949 & 1.57728 & 1.57997 \\
Test Error (\%) & 1.65747 & 1.44154 & 1.77011 & 1.79838 & 1.85429 \\
Max Training Error (\%) & 3.38446 & 3.87043 & 2.11045 & 8.31046 & 5.54259 \\
Max Test Error (\%) & 5.33410 & 3.80190 & 4.87684 & 5.93603 & 4.28629 \\
${ }^{*}{ }^{2}$ & 0.99845 & 0.99811 & 0.99829 & 0.99531 & 0.99729 \\
${ }^{*}$ CCo & 0.99923 & 0.99906 & 0.99914 & 0.99765 & 0.99865 \\
${ }^{*}$ CoE & 0.99843 & 0.99785 & 0.99822 & 0.99506 & 0.99725 \\
${ }^{*}$ GoF(\%) & 96.0383 & 95.3617 & 95.7758 & 92.9712 & 94.7575 \\
${ }^{*}$ CoD & 0.99845 & 0.99811 & 0.99829 & 0.99531 & 0.99729 \\
\hline
\end{tabular}

* these statistical criteria are defined in Supporting Information.

\subsection{Visualization of unexplained molecular features of OBCs}

As mentioned earlier, application of developed hybrid stochastic model enables patterning non-linear behavior of polymerization reactions considering that only little is known about mechanistic aspects of chain shuttling reactions. Their complex nature makes a deeper understanding of the combined effects of dual catalyst system and CSA on microstructural and architectural characteristics of OBCs more complicated.

One unique feature of combining ANN with KMC is that it allows for construction of contour plots of selected microstructural features (consider contour plot of Figure 3A for instance). As described in detail in our first paper on $O B C$ [12], even for a recipe with $10^{11}$ molecules fed into the simulation volume, KMC simulator needs a very long time to be run (10h). Thus, for finding recipes with a defined $\mathrm{OBC}$-microstructure, the use of $\mathrm{KMC}$ alone makes determining the parameters for a recipe with the desired microstructure impossible. The use of artificial intelligence, however, with the ability of learning, generalizing, and decision-making, significantly reduces the calculation time and by limiting the number of use of KMC simulations to a reasonably sized array of recipes to be virtually synthesized in KMC. Notably, 5 contour plots (one for each monomer ratio tested) with such detailed information has been constructed 
using $5 \times 40000$ recipes that were received by ANN, while only $175 \mathrm{KMC}$-simulations were used as input data using 5 different monomer ratios.

Setting up the ANN only took about $85 \mathrm{~min}$, including determining the data for the abovementioned contour-plots, consisting of 5 different monomer ratios and 5 different parameters $\left(\overline{\mathbf{D P}}_{\mathbf{n}}^{\text {SOFT }}, \overline{\mathbf{D P}} \mathbf{n}_{\mathbf{H A R D}}, \overline{\mathbf{E S L}}{ }^{\text {SOFT }}, \overline{\mathbf{E S L}}{ }^{\text {HARD }}\right.$, and LP), i.e. in total $5 \times 5 \times 40000$ output data points. The ANN has been developed in-house comprising about 16000 code lines.

The power of the approach of hybridizing KMC and ANN can be understood when comparing the calculation time of KMC (ca. 10 hours per recipe) with the time to build and evaluate the ANN (ca. 85 minutes). Hence, once a sufficient number of systematically KMC-simulations are fed into the ANN, one can very accurately predict a whole range of scenarios by the ANN instead of making another time-consuming KMC calculation. If one would calculate the 40000 output scenarios that were predicted by ANN by KMC instead, the required calculation time would be ca. 45 years. Hence, a "phase diagram" can be determined in a rather straightforward fashion with KMC-ANN, which is not possible easily using KMC alone due to the sheer number of scenarios required to be calculated.

Although this scenario is unrealistic (calculation of 40000 scenarios for 45 years without some kind of manual optimization), it becomes immediately apparent that hybridizing KMC and ANN is the by far superior approach.

Figure 3 and Figure 4 show bivariate contour plots of $\overline{\mathrm{DP}}_{\mathrm{n}}^{\mathrm{SOFT}}$ and $\overline{\mathrm{DP}}_{\mathrm{n}}^{\mathrm{HARD}}$ in terms of CC and $\log \left(\mathrm{CSA}\right.$ Level) for cases with constant MR values, respectively. In case of $\overline{\mathrm{DP}}_{\mathrm{n}}^{\mathrm{SOFT}}$, presented in Figure 3, an increase of CSA concentration at given MR and CC values yields OBCs with shorter block lengths. A similar trend was seen for $\overline{\mathrm{DP}}_{\mathrm{n}}^{\mathrm{HARD}}$ in Figure 3, which can be expected from the chain shuttling concept [1-3]. On the other hand, the monomer ratio MR solely governs the variation pattern of $\overline{\mathrm{DP}}_{\mathrm{n}}^{\mathrm{SOFT}}$. It should be mentioned that higher MR values, in view of very higher reactivity ratio of ethylene compared to 1-octene in case of Catalyst 2, can upset the balance between self- and cross-shuttling reactions toward lengthening of soft blocks [12]. Thus, it can be concluded that the length of soft blocks is comparatively more sensitive to the 
chain shuttling reaction parameters. This is underlined by the fact that values of this quantity change between 15 and 3620 (Figure 3) under applied operating condition, while those of $\overline{\mathrm{DP}}_{\mathrm{n}}^{\mathrm{HARD}}$ vary from 100 to 3720 (Figure 4), irrespective of the MR value. Bearing in mind that shuttling takes place regardless of catalyst type, a further reason for sensitivity of $\overline{\mathrm{DP}}_{\mathrm{n}}^{\mathrm{SOFT}}$ to MR value is that the rate constant for "transfer to hydrogen" is 100 times higher in case of Catalyst 1 [12]. Therefore, under batch operation conditions, the hydrogen concentration drops with polymerization time, which mainly affects soft segments due to their higher tendency to hydrogen abstraction.

In a similar way, for a constant CSA concentration, the CC has a very weak influence on the $\overline{\mathrm{DP}}_{\mathrm{n}}^{\mathrm{HARD}}$, but does in turn influence the $\overline{\mathrm{DP}}_{\mathrm{n}}^{\mathrm{SOFT}}$. For low level of CSA, the length of the soft block increases with increasing CC (quantity of the Hafnium based catalyst leading to the soft segments), whatever the MR. This is also the case for high level of CSA and high MR. For low MR (and high level of CSA) the length of the soft block does decrease. This may be attributed to the reactivity ratio of 1-octene, which is low. There are two reasons why a high quantity of 1-octene assists in reduction of $\overline{\mathrm{DP}}_{\mathrm{n}}^{\mathrm{SOFT}}$. An abundance of 1-octene in the reaction media leads to formation of soft blocks ending in 1-octene. As in Table 1, rate of ethylene homopolymerization by Cat 1 is 100 times higher than that of 1-octene. Hence, on average, a lower activity of this catalyst is probable. Moreover, transfer to hydrogen and shuttling to CSA together ease formation of dormant chins, so that chains have a very limited chance of growth. As a result, formation of chains with shorter $\overline{\mathrm{DP}}_{\mathrm{n}}^{\mathrm{SOFT}}$ would be expected.

An interesting consequence of these findings is that one can control / play on the size of the soft blocks by varying the CC and/or the MR without affecting significantly the length of the hard block. This is of particular interest in terms of materials design considering the complexity of the system. The length of the hard block can in turn be adjusted using the quantity of chain shuttling agent in the reactive medium. 
In addition, the width of the soft block length distribution could be cogently compared to that of the hard segments. Three-dimensional representation of $\overline{\mathrm{DP}}_{\mathrm{n}}^{\mathrm{SOFT}}$ and $\overline{\mathrm{DP}}_{\mathrm{n}}^{\mathrm{HARD}}$ in terms of operating condition are given in SI (Figure S1 and Figure S2).

The trained ANN (using the aforedescribed 175 KMC-scenarios) can be used to predict the parameters necessary for producing OBCs with predetermined microstructural features. Hence, it is an unexpected, at the same time interesting, signature of chain shuttling reaction, which has been uncovered for the first time in this work.

The possibility of careful selection and tuning of the polymerization conditions by the developed model enables developing OBCs with desired hard and soft block length, allowing for manufacturing special grades of OBCs. The reader is referred to Figure S3 and Figure S4, respectively, in the SI to evaluate possibility of fine-tuning In case of $\overline{\mathrm{DP}}_{\mathrm{n}}^{\mathrm{SOFT}}$ and $\overline{\mathrm{DP}}_{\mathrm{n}}^{\mathrm{HARD}}$.

An almost similar behavior, but with more complexities, is seen for variation of the ethylene sequence lengths $\overline{\mathrm{ESL}} \mathrm{SOFT}$ and $\overline{\mathrm{ESL}} \mathrm{HARD}$ in terms of CC and $\log$ (CSA Level) for cases with constant MR values (Figure 5 and Figure 6). The major difference between these microstructural characteristics, however, is higher sensitivity of $\overline{\mathrm{ESL}}$ to operating conditions with respect to $\overline{\mathrm{DP}}_{\mathrm{n}}$, which was expected. The corresponding three-dimensional plots of $\overline{\mathrm{ESL}}$ SOFT and $\overline{\text { ESL }} \mathrm{HARD}$ are given as Figure S5 and Figure S6 in SI, respectively. The incorporation of 1-octene comonomer to hard and soft blocks determines the $\overline{\mathrm{ESL}}$ of hard and soft block of OBCs. It is obvious that the variation in instantaneous incorporation of comonomers into the blocks is much higher compared to variation in a given OBC chain.

From a probability perspective, either population or distribution of comonomers in the blocks justifies dissimilarities in $\overline{\mathrm{ESL}}$ contour plots compared to $\overline{\mathrm{DP}}_{\mathrm{n}}$ moving from low to high MR values. Once again, however, the sensitivity of $\overline{\mathrm{ESL}} \mathrm{SOFT}$ to the operating conditions is more pronounced, which can be explained by the poor 1-octene incorporatability of Catalyst 1 . In agreement with this, the model suggests two CSA levels for a given MR and CC to obtain OBCs with preset $\overline{\mathrm{ESL}} \mathrm{SOFT}$ of 3, 24, and 48 (see Figure S7 in SI). Moreover, $\overline{\mathrm{ESL}}$ is strongly dependent on the concentration of ethylene in the reaction media, which can be seen from the serious 
variation of both $\overline{\mathrm{ESL}}$ SOFT and $\overline{\mathrm{ESL}}{ }^{\mathrm{HARD}}$ plots with MR (Figure S7 and Figure S8 in SI). These results can be considered as a new challenge/opportunity for those experimentally working on chain shuttling polymerization. 
(A)

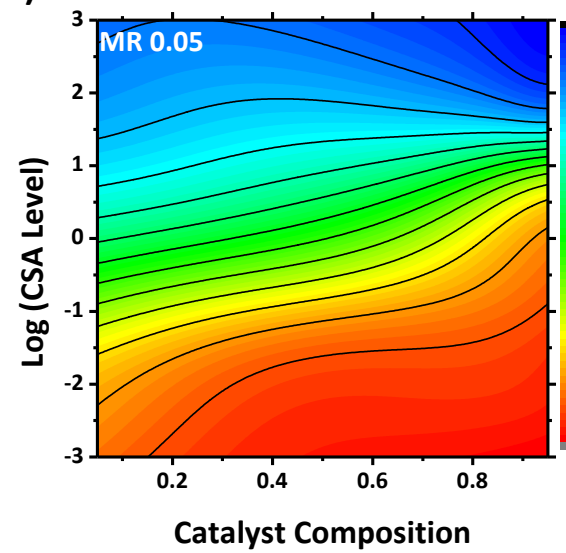

(C)

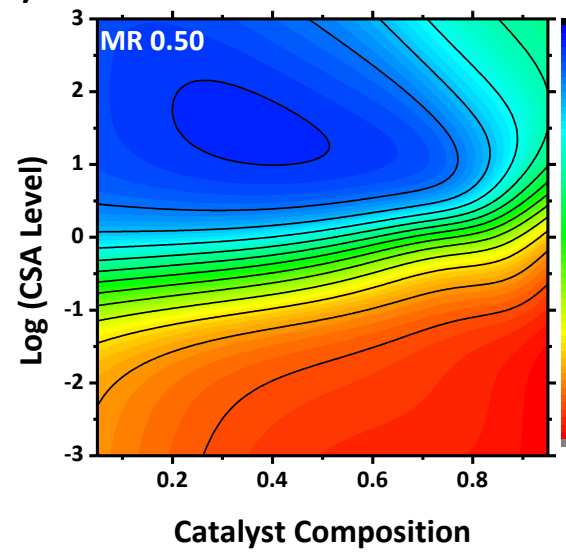

(E)

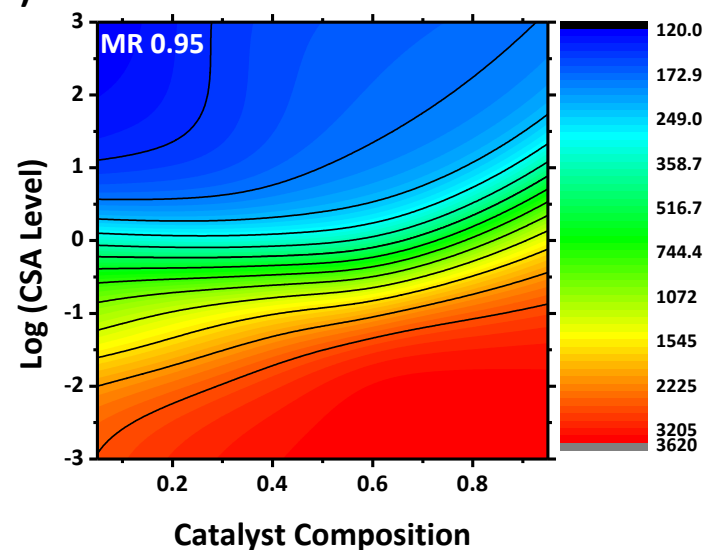

(B)

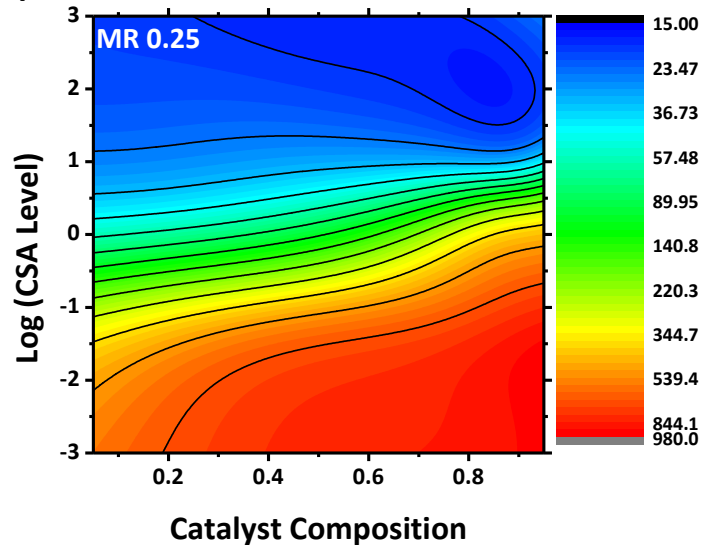

(D)
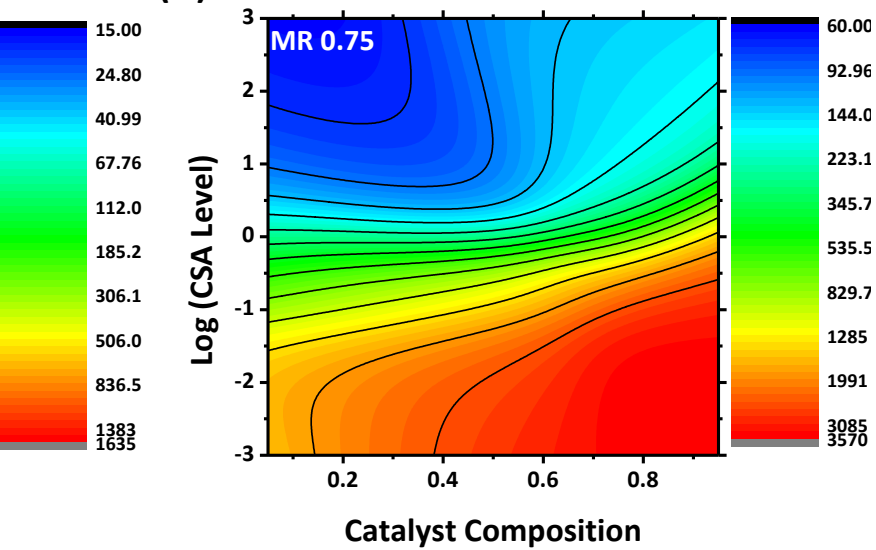

Figure 3. Bivariate contour plots of $\overline{D P}_{\mathbf{n}}^{\text {SOFT }}$ in terms of CC and $\log (\mathrm{CSA}$ level) for cases with constant $M R$ values for (A) $M R=0.05$, (B) $M R=0.25$, (C) $M R=0.50$, (D) $M R=0.75$, and (E) $M R=0.95$. 
(A)

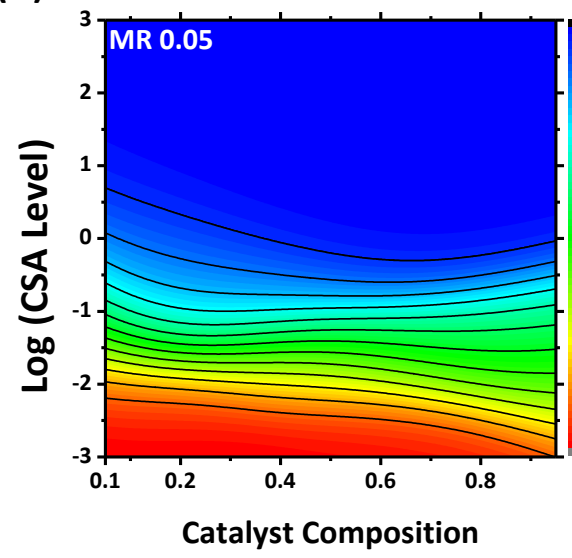

(C)

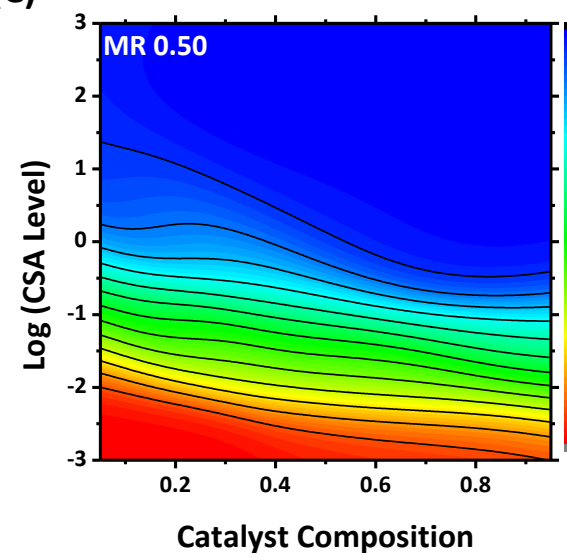

(E)

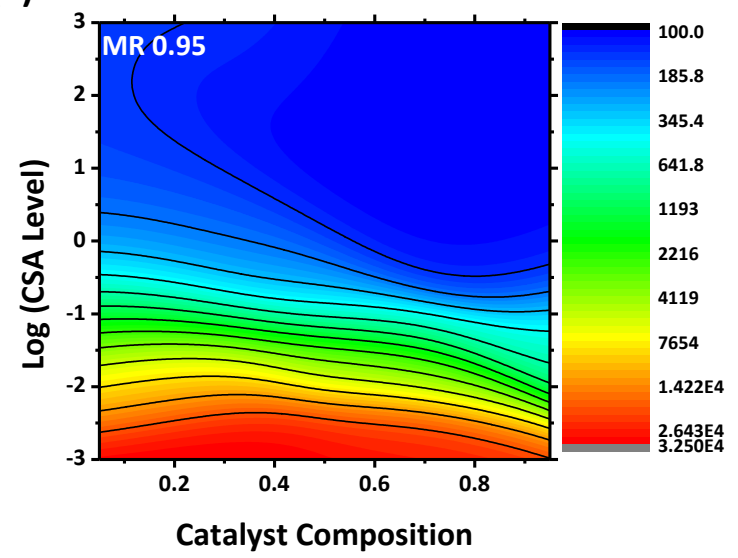

(B)
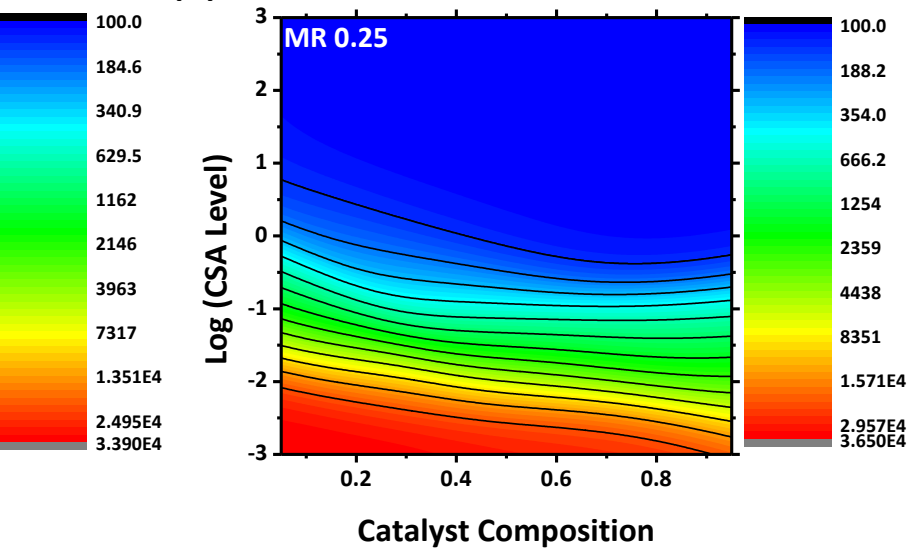

(D)
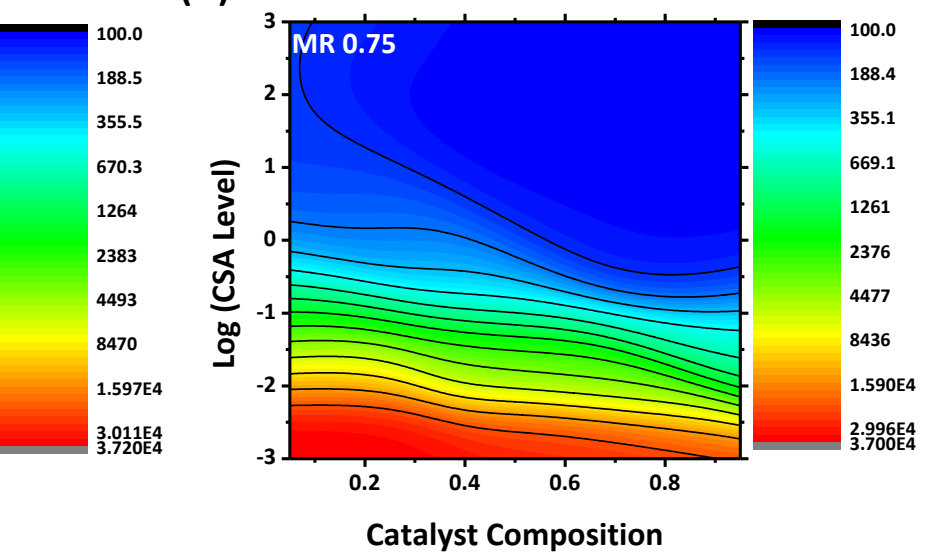

Figure 4. Bivariate contour plots of $\overline{D P}_{n}^{\text {HARD }}$ in terms of CC and $\log (C S A$ Level) for cases with constant $M R$ values for (A) $M R=0.05$, (B) $M R=0.25$, (C) $M R=0.50$, (D) $M R=0.75$, and (E) $M R=0.95$. 
(A)

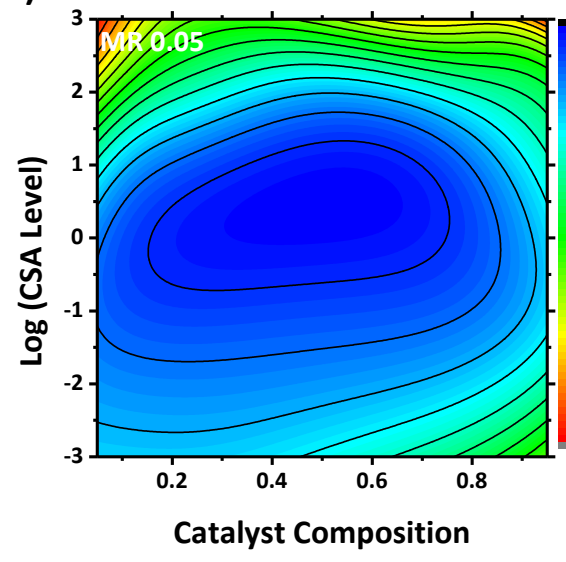

(C)

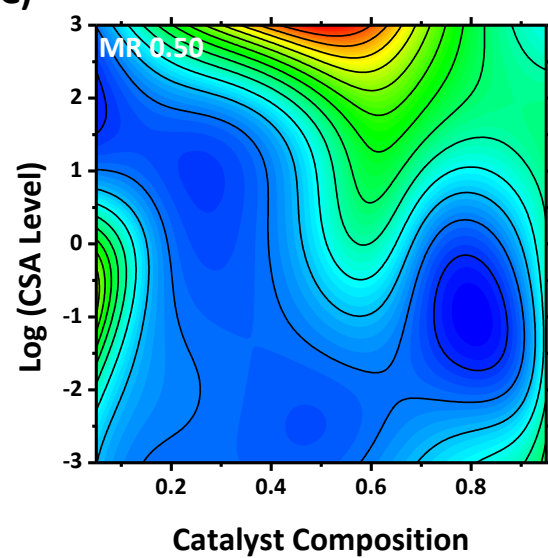

(E)

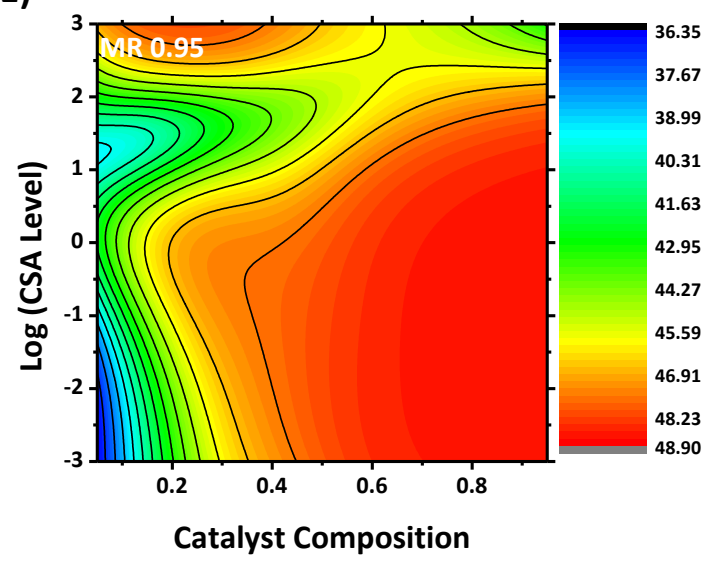

(B)
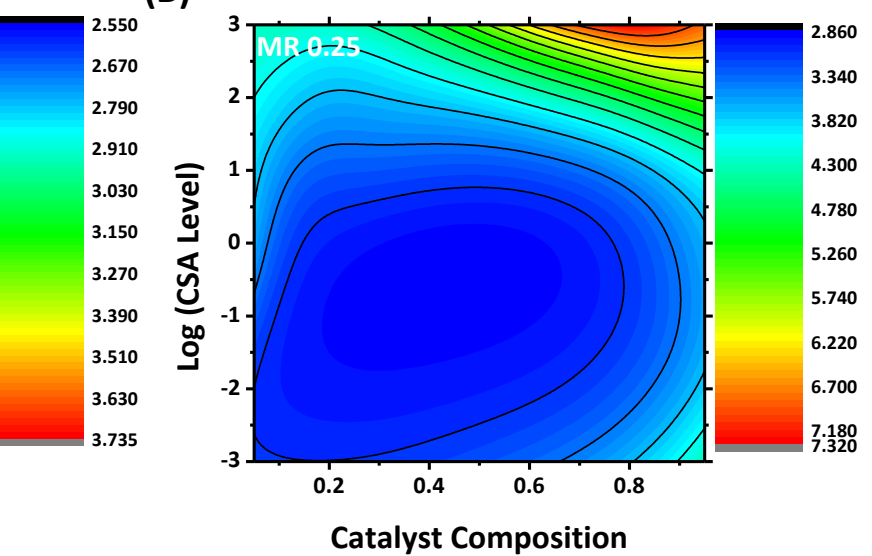

(D)
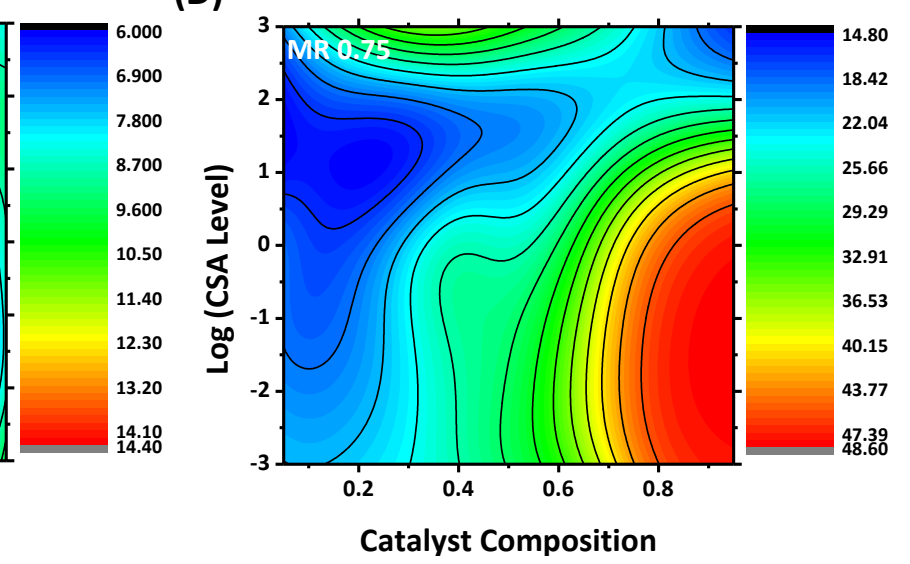

Figure 5. Bivariate contour plots of $\overline{\mathrm{ESL}}$ SOFT in terms of CC and $\log (\mathrm{CSA}$ Level) for cases with constant $M R$ values for (A) $M R=0.05$, (B) $M R=0.25$, (C) $M R=0.50$, (D) $M R=0.75$, and (E) $\mathrm{MR}=0.95$. 
(A)

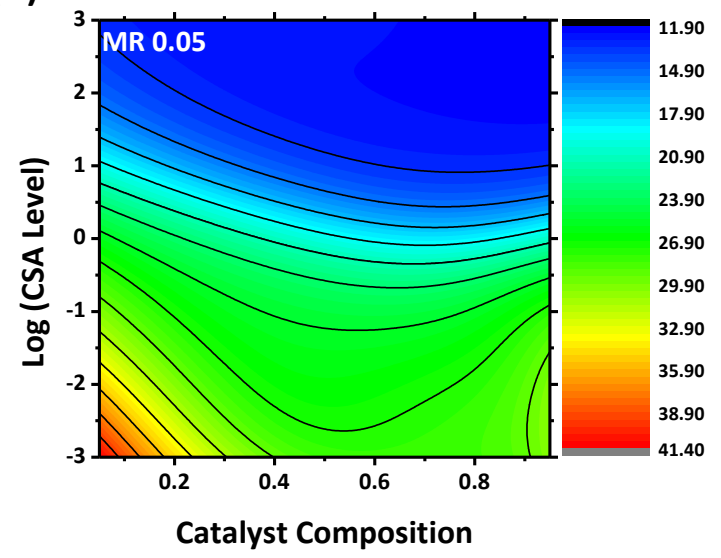

(C)

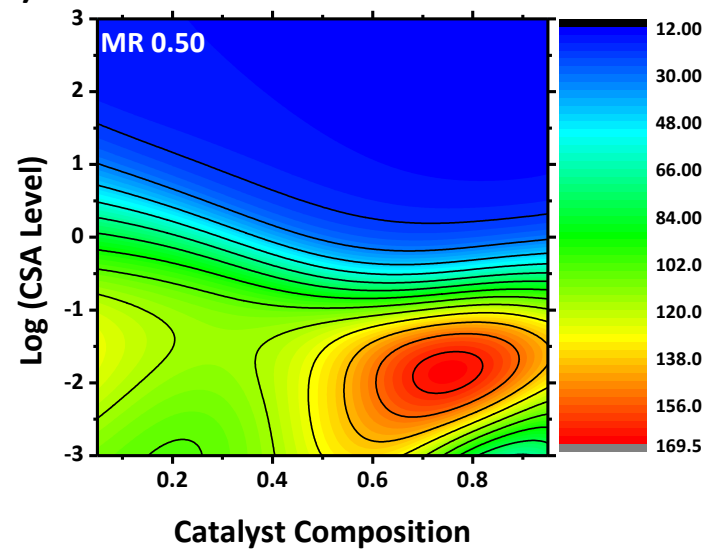

(E)

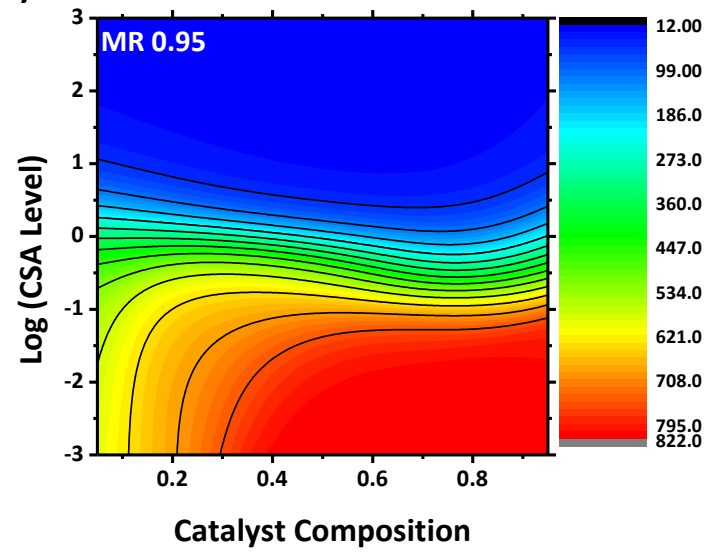

(B)

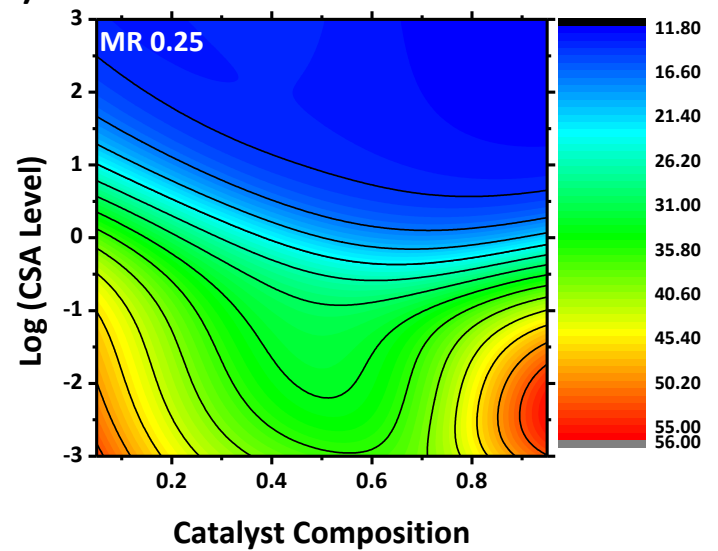

(D)

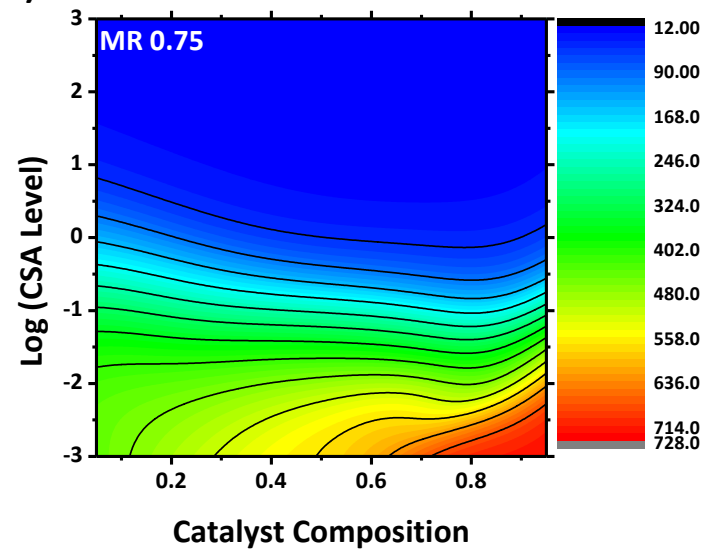

Figure 6. Bivariate contour plots of $\overline{\mathrm{ESL}}{ }^{\mathrm{HARD}}$ in terms of CC and $\log (\mathrm{CSA}$ Level) for cases with constant $M R$ values for (A) $M R=0.05$, (B) $M R=0.25$, (C) $M R=0.50$, (D) $M R=0.75$, and (E) $M R=0.95$. 
Figure 7 shows bivariate contour plots of LP in terms of CC and log (CSA Level) for cases with constant MR values. As can be seen, MR value has a little effect on the distribution of LP. This is because the multi-block nature of produced copolymers is mainly governed by the CSA Level. For any given CC value, an increase of CSA Level facilitates shuttling reaction and, consequently, OBCs with more blocks are produced. Each contour plot in Figure 7 demonstrates that OBCs with LP values less than unity are born (blue and black areas). This is the case where concentration of CSA is very low and we have a mixture of unshuttled hard and soft homopolymers and shuttled multi-block copolymer chains. Accordingly, operating conditions needed for developing OBCs with different blocky nature are identified. The reader is referred to see Figure S9 in SI for a three-dimensional view of LP variation.

The plots have to be understood in the fashion that the blue shaded areas correspond to low numbers of blocks and thus essentially reactor blends of high and low comonomer content random copolymers along with few di- or tri-block copolymers, while the red areas refer to block-copolymers with so many blocks that they can almost considered to be random copolymers, as each block is rather short.

It is obvious and easily understandable that the main influence factor on LP is the $\log$ (CSA level), but it is also clear that the influence is more complex, as the "height lines" are not only horizontal as would be expected from a simple 1:1 relation. Instead it is obvious that, especially at higher MR, high catalyst compositions (i.e. higher amounts of the readily comonomer incorporating catalyst) lead to a significantly lower LP in comparison to low CC. This can be understood in an easier way looking at Figure 7F, which shows LP as a function of CSA-level averaged for all CC and MR. the black squares stand for the average of the data, while red and green triangles represent the minimum and maximum values found, respectively. Additionally the dynamic range (ratio of highest and lowest value in Fig. S10, blue stars) is given on the right $\mathrm{y}$-axis. The complete data are given in Figure $\mathbf{S 1 0}$ in SI. As can be seen, a wide range of products with different LP values can be produced at different CSA concentrations, however, variation patterns are completely changed moving from cases with low CSA concentration to ones with very high CSA contents. The complexity of shuttling reactions under the influence of each 
parameter, alone or in association with other operating factors, may still necessitate visualization of some more aspects in terms of operating conditions, which is possible armed with developed KMC-ANN tool.

(A)

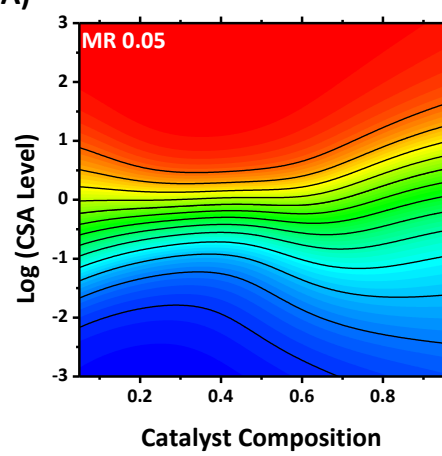

(C)

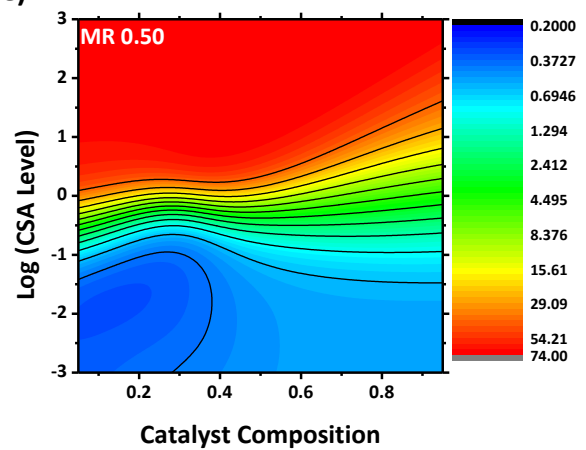

(E)

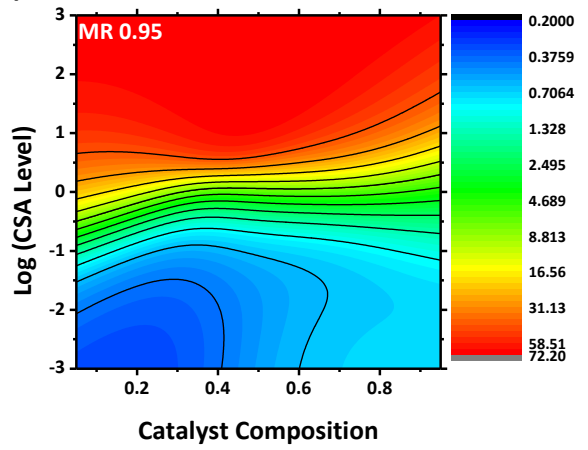

(B)

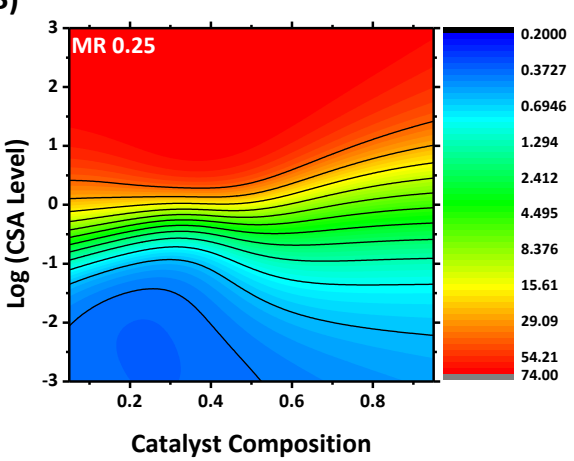

(D)
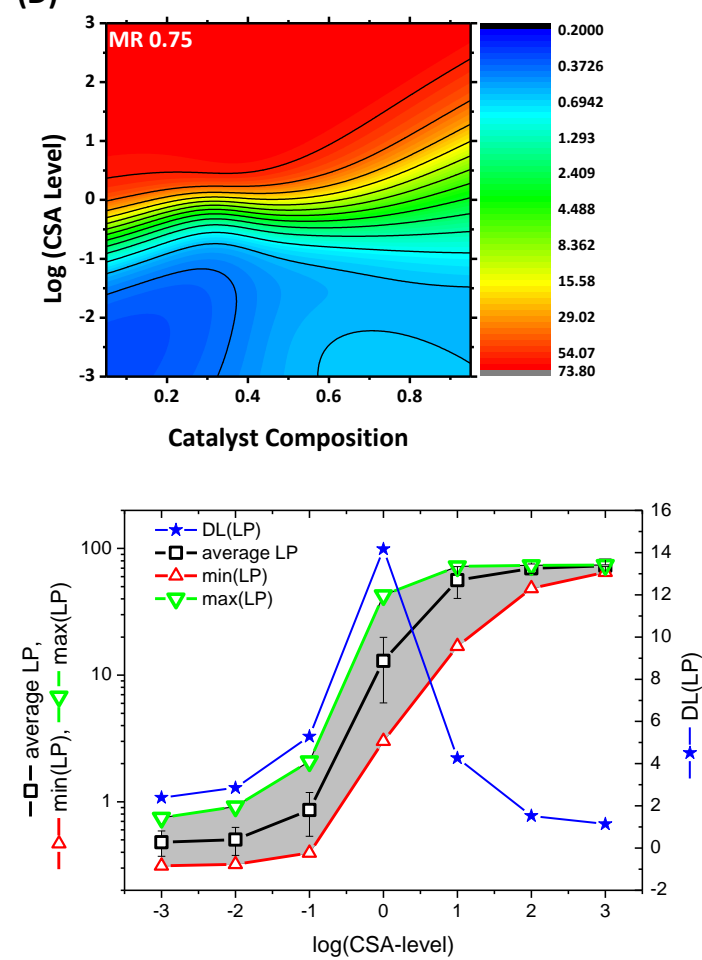

Figure 7. Bivariate contour plots of LP in terms of CC and $\log$ (CSA Level) for cases with constant $M R$ values for (A) $M R=0.05$, (B) $M R=0.25$, (C) $M R=0.50$, (D) $M R=0.75$, and (E) $M R=0.95$. (F) $L P$ as a function of CSA-level (averaged for $M R=0.05-0.95$ and $C C=0.05-0.95$. The error bars were calculated from the standard deviation of LP. 
It is remarkable that the CSA-level dominates LP for most $\log (\mathrm{CSA})$-levels tested so much that the relative standard deviation for $\log (\mathrm{CSA})=-3,-2,+2$, and +2 is below $25 \%$ and the dynamic range is below 3 , i.e. LP is almost independent of the other parameters. Only for $\log (\mathrm{CSA})=-1,0$, and +1 a sizable influence of CC and MR can be observed. In other words, if $\log (\mathrm{CSA})$ is either rather high or rather low, LP is almost exclusively controlled by $\log (\mathrm{CSA})$, while at intermediate $\log (\mathrm{CSA})$-level, MR and CC play a significant role as well.

Figure 8 demonstrates the behavior of chain shuttling reaction through possibility analysis of producing OBCs with predetermined LP in terms of CC and log (CSA Level) for cases with constant MR values. Careful selection of cases with LP values of $0.5,1,2,5$, and 50 uncovered the potential of system at any given operating condition to yield multi-block copolymers and, furthermore, shows that the $\log (\mathrm{CSA}$ Level) is the key parameter determining LP. Since each OBC shows a special thermoplastic elastomer behavior, such controlled OBCs with specified blockiness can meet many industrial requirements.

For instance, the first case $(\mathrm{LP}=0.5)$ is an $\mathrm{OBC}$ with, on average, $50 \%$ of hard and soft homopolymers and $50 \%$ of di-block copolymer chains (Figure $\mathbf{8 A}$ ). This is the only case among all in Figure 8 that such OBCs with low degree of blockiness are producible at certain (low) CC and MR levels. If the concentration of ethylene is high (corresponding to plots assigned to MR values of $0.5,0.75$, and 0.95 in Figure $8 \mathrm{~A}$ ), OBCs with LP of 0.5 cannot be produced at CC values greater than 0.5 , which is understandable in view of very low possibility of chain transfer to Catalyst 2 (producing hard blocks) at such low concentration of CSA[10].

Nonetheless, for $\mathrm{LP}=1-50$ (from Figure $8 \mathrm{~B}-\mathrm{E}$ ), shuttling reaction is mostly governed by the $\log ($ CSA Level) and, consequently, the system allows producing of a copolymer without any limitation in operating condition due to CC or MR. Hence, production of OBCs with higher number of blocks was found at higher CSA levels, as signaled by a shift in curves moving from Figure $8 \mathrm{~A}$ to Figure $8 \mathrm{E}$. Figure $8 \mathrm{~A}$ shows which operating conditions are necessary for $\mathrm{LP}=0.5$, i.e. more or less random copolymers ( $\mathrm{LP}=0.5$ means on average 0.5 block junctions per chain). Di- (Figure 8B) and triblock copolymers (Figure 8C) on average can be produced as well as typical 
multi-block copolymers (LP=5 (Figure 8D) and LP=50 (Figure 8E)), which represent blocky and almost random block copolymers. Hence, it is possible to design the blockiness of the desired copolymer within a very broad range. It is clear additionally that LP is mostly dependent on $\log ($ CSA-level), which is expected and that MR and CC do not play a major role as long as LP>2 (Figure 8C-E).

Hence, without doubt, developed code can precisely pattern possibility of producing copolymer chains with higher LP value, depending on which LP would practically be considered to be assigned to an ideal multi-block microstructure.

The finding that $\mathrm{LP}<1$ is only possible for low $\mathrm{CC}$ is indicative of the different nature of the catalysts. For further understanding of this case, reader is referred to Figure 7F and to SI (Figure S10).

Eventually, it should be pointed out that the variations of the curves in Fig. 8 are mostly systematic as a function of MR. Considering that the data in Fig. 8 are derived quantities, which are not the direct result of the ANN, the systematic trends show the high reliability of the KMCANN approach. 

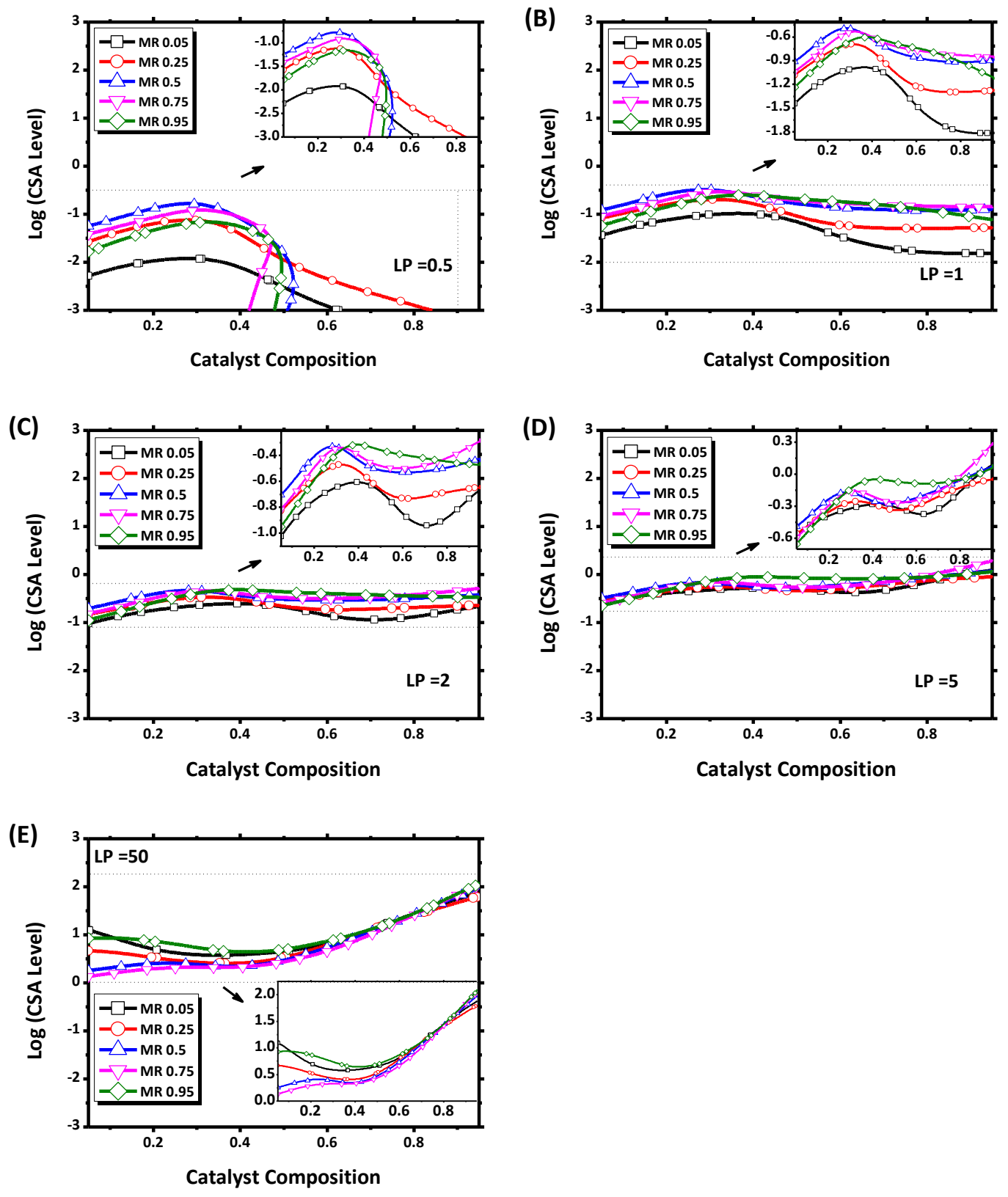

Figure 8. Possibility analysis and operating conditions for producing $\mathrm{OBCs}$ with predetermined blockiness (LP). (A) $L P=0.5$, (B) $L P=1$, (C) $L P=2$, (D) $L P=5$, and (E) $L P=50$.

\subsection{Production of tailor-made OBCs applying microstructural master plots}

In this section, we provide some typical results among all possible cases obtained by the hybrid model, to give access to tailor-made OBCs. Figure 9 illustrates that it is possible with the aid of the dynamic hybrid model developed in this work to collect detailed information about 
$\mathrm{LP}, \overline{\mathrm{DP}}_{\mathrm{n}}^{\mathrm{HARD}}, \overline{\mathrm{DP}}_{\mathrm{n}}^{\mathrm{SOFT}}, \overline{\mathrm{ESL}}{ }^{\mathrm{HARD}}$, and $\overline{\mathrm{ESL}}$ SOFT of OBCs at any given operating condition. These master plots correspond to scenarios 59 and 129 synthesized by the KMC simulator (Table 2).

Table 4 gives detailed information about the specifications of tailored polymer, which are marked in master plots of Figure 9, and compares the values of microstructural features for scenarios 59 (a) and 129 (b), which differ in terms of monomer ratio. In case of each molecular characteristic, the difference between the lowest value (LV) and highest value (HV) of the assigned quantity is of critical importance, for it signifies maximum deviation in that quantity. The relative error in prediction of target macromolecular feature is defined as the quotient of the absolute difference between ANN model and KMC simulator values to the absolute maximum deviation ( $\mid$ HV-LV $\mid$ ) and expressed in percent. From the error, given in percent, one can conclude that values predicted by the hybrid model are very close to those obtained by KMC simulator at identical operating condition. Despite the fact that | HV-LV | quantity is generally very large; errors in prediction of microstructural characteristics of different $O B C$ grades are negligible. The maximum deviation is overall less than $1 \%$. In addition to the fact that Figure 9 and Table 4 prove the accuracy of model, they can be considered as blueprints in tailoring OBCs. Thus, one can simply place a ruler on the provided master plots and draw straight vertical or horizontal lines to predict the microstructural aspects of the envisioned OBCs at certain operating conditions. This is a very simple way of classifying OBCs into different grades and tailoring their microstructure. 
(A)
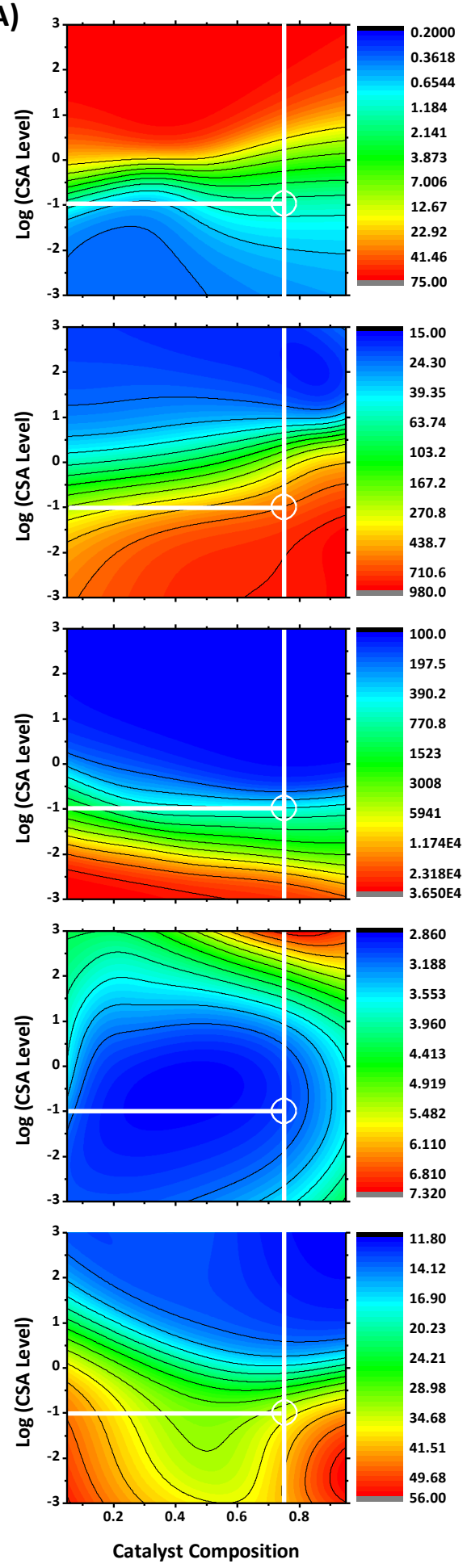

(B)
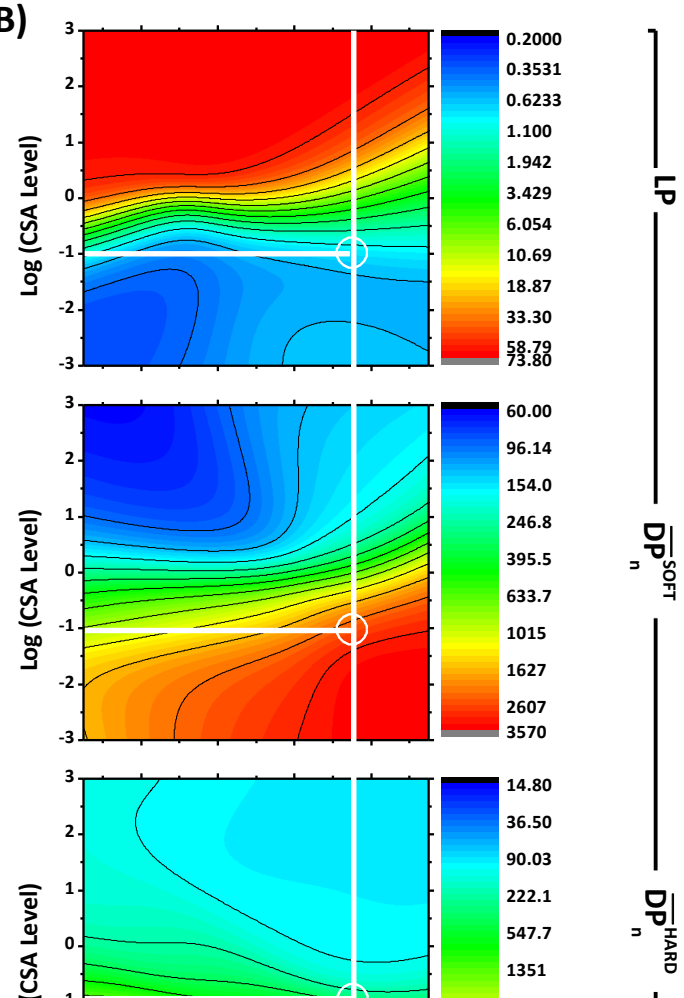

อัง
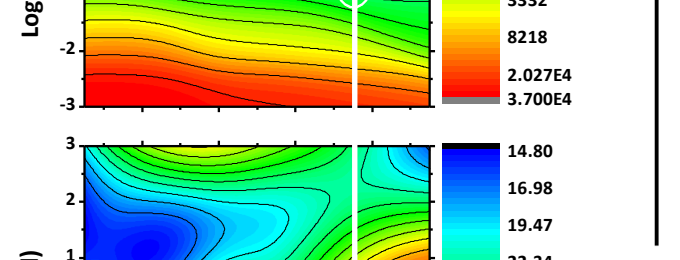

界|
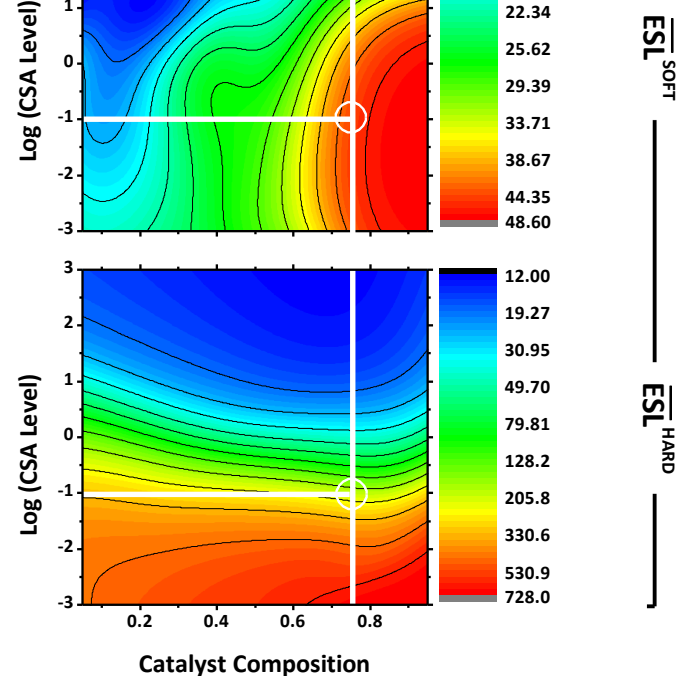

Figure 9. Typical master plots obtained by the hybrid model for tailoring OBCs. A) Scenario 59, b) Scenario 129. 
Table 4. Predictability of hybrid models toward scenarios 59 and 129 synthesized by KMC simulator (Table 1).

Catalyst Composition: $0.75 ; \log ($ CSA Level): -1

\begin{tabular}{|c|c|c|c|c|c|c|c|}
\hline & Scenario & $\begin{array}{l}\text { Monomer } \\
\text { Molar } \\
\text { Ratio }\end{array}$ & $\begin{array}{l}\mathrm{Y} 1 \\
\left(\overline{\mathrm{DP}}_{\mathrm{n}}^{\text {SOFT }}\right)\end{array}$ & $\begin{array}{l}\mathrm{Y} 2 \\
\left(\overline{\mathrm{DP}}_{\mathrm{n}}^{\text {HARD }}\right)\end{array}$ & $\begin{array}{l}\text { Y3 } \\
\left(\overline{E S L} \text { soFT }^{\prime}\right)\end{array}$ & $\begin{array}{l}\text { Y4 } \\
\left(\overline{E S L}{ }^{\text {HARD }}\right)\end{array}$ & $\begin{array}{l}\text { Y5 } \\
\text { (LP) }\end{array}$ \\
\hline \multirow[t]{2}{*}{ ANN } & 59 & 0.25 & 614.87 & 528.64 & 3.05 & 35.02 & 1.35 \\
\hline & 129 & 0.75 & 2480.81 & 401.33 & 44.36 & 203.79 & 0.79 \\
\hline \multirow[t]{2}{*}{ KMC } & 59 & 0.25 & 591.81 & 450.23 & 2.98 & 37.05 & 0.84 \\
\hline & 129 & 0.75 & 2173.81 & 432.77 & 42.43 & 245.63 & 0.84 \\
\hline LV & & - & 5.13 & 7.12 & 2.34 & 2.19 & 0.00 \\
\hline HL & & - & 38085.60 & 3736.87 & 48.97 & 825.43 & 74.28 \\
\hline Error & 59 & 0.25 & 0.06 & 2.10 & 0.14 & 0.24 & 0.67 \\
\hline (\%) & 129 & 0.75 & 0.81 & 0.84 & 4.12 & 5.08 & 0.07 \\
\hline
\end{tabular}

\subsection{Classification of the microstructural landscape of OBCs}

On the basis of non-linear complex nature of chain shuttling reaction [4], a very small change in operating condition may lead to a serious deviation from a multi-block copolymer. Through a detailed view of molecular landscape of OBCs with different degree of regulation of $\overline{\mathrm{ESL}}$ and $\overline{\mathrm{DP}}_{\mathrm{n}}$, one can correlate operating conditions with molecular features of the resulting copolymers. Here we attempt to classify OBCs into distinct categories, which can be used for a better understanding, providing polymer reaction engineers with an opportunity to obtain an overview of the molecular landscape of OBCs, thereby shedding more light on mechanistic details of chain shuttling polymerization. Each molecular landscape specifies CC, CSA and MR values necessary for synthesizing a very unique OBC.

For example, the results presented in this section challenge the concept that soft segments always have a smaller sequence length due to good comonomer incorporation. This type of molecular architecture is known as standard $\mathrm{OBC}$ and firstly introduced and commercialized by the DOW group [1]. We identified by the aid of our hybrid model that one can supply operating conditions at which the comonomer incorporation potential of the catalyst species assumes an inverse trend. Accordingly, we introduce for the first time operating conditions required to 
synthesize new grades of OBCs with different natures, among which is the case with longer ethylene sequences in soft blocks. Moreover, we introduce a very special grade of $\mathrm{OBC}$, namely twin-block OBC (TB-OBC) which appears like a homopolymers with similar blocks.

As a first kind of special OBCs introduced in this work, operating conditions that enable synthesizing OBCs with $\overline{\mathrm{DP}}_{\mathrm{n}}^{\mathrm{HARD}}=\overline{\mathrm{DP}}_{\mathrm{n}}^{\mathrm{SOFT}}$ are established. Figure $\mathbf{S 1 1}$ in $\mathrm{SI}$ visualizes the possibility of producing such special grades of $\mathrm{OBC}$ through three-dimensional representation with color guide showing distribution of length of hard and soft blocks. In order to provide an easily understandable representation of these admittedly rather complex results, Figure 10

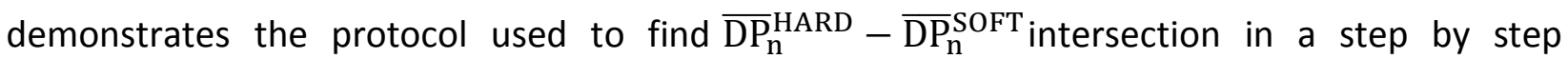
manner.

The top row of Figure 10 gives a 3D-view of $\overline{\mathrm{DP}}_{\mathrm{n}}^{\mathrm{HARD}}$ and $\overline{\mathrm{DP}}_{\mathrm{n}}^{\text {SOFT }}$ as a function of $\log (\mathrm{CSA}$ Level) and CC. As a next step (middle row), the intersection $\left(\overline{\mathrm{DP}}_{\mathrm{n}}^{\mathrm{HARD}}=\overline{\mathrm{DP}}_{\mathrm{n}}^{\text {SOFT }}\right)$ is determined and marked by the thick black line. The red area denotes $\overline{D P}_{n}^{\text {HARD }}>\overline{\mathrm{DP}}_{\mathrm{n}}^{\text {SOFT }}$, while green denotes $\overline{\mathrm{DP}}_{\mathrm{n}}^{\mathrm{HARD}}<\overline{\mathrm{DP}}_{\mathrm{n}}^{\mathrm{SOFT}}$. The last figure shows the variation of $\overline{\mathrm{DP}}_{\mathrm{n}}^{\mathrm{HARD}}=\overline{\mathrm{DP}}_{\mathrm{n}}^{\mathrm{SOFT}}$ as a function of MR.

This is a very unique view of chain shuttling hidden face that has been disclosed for the first time. Of note, the hybrid model can also identify operating conditions for producing OBCs with any sort of relationship between hard and soft block length, e.g. $\overline{\mathrm{DP}}_{\mathrm{n}}^{\mathrm{HARD}}=3 \overline{\mathrm{DP}}_{\mathrm{n}}^{\mathrm{SOFT}}$. 

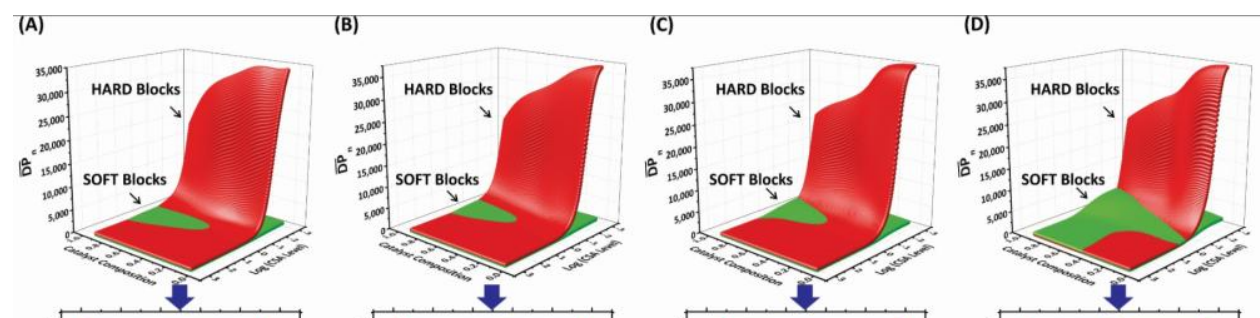

(E)
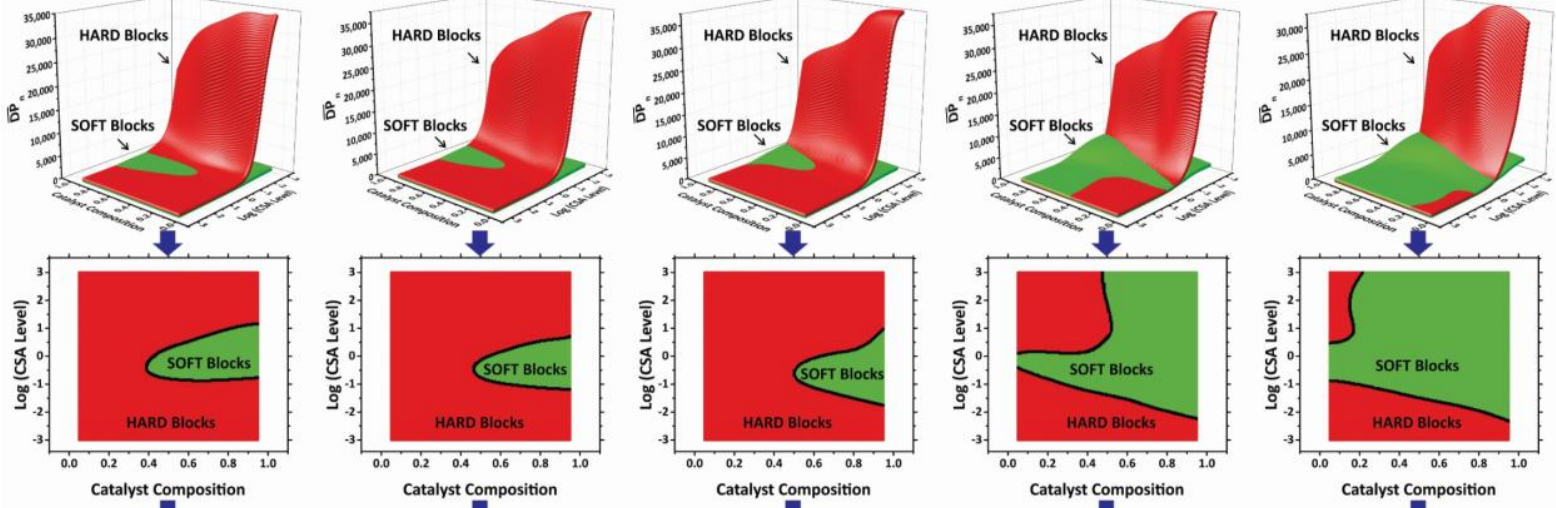

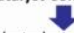
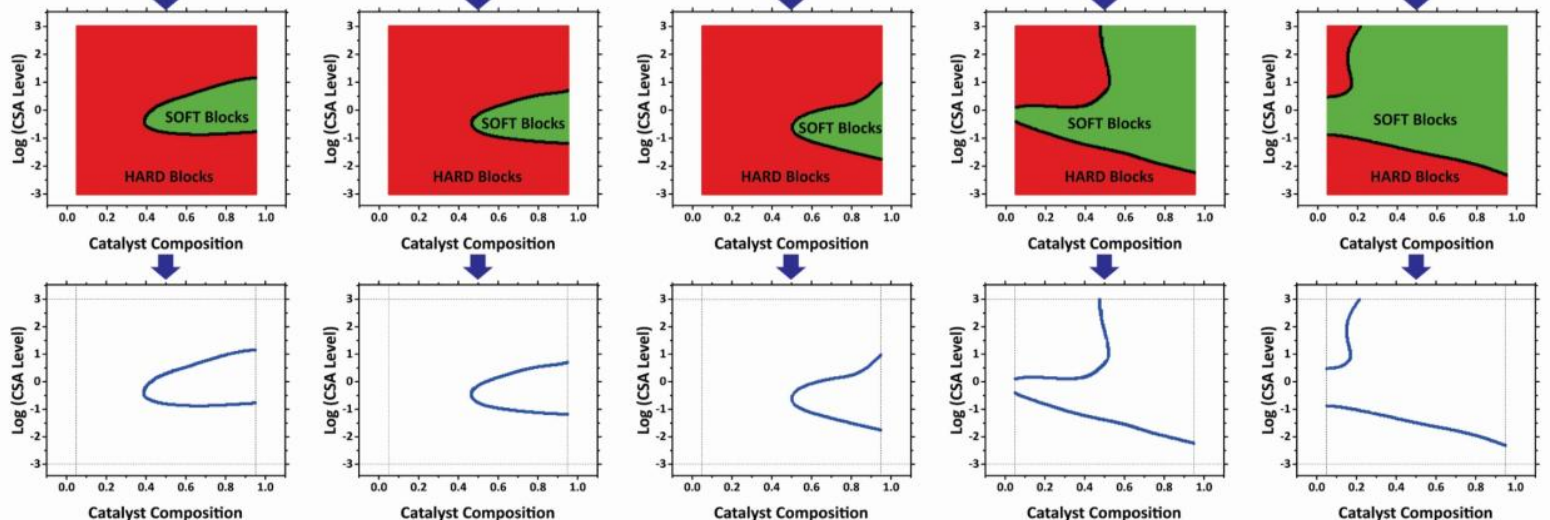

Catalyst Composition

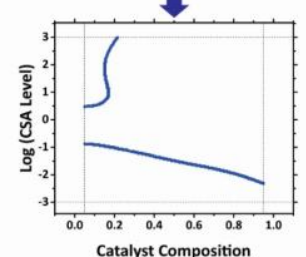

Figure 10. Possibility analysis of obtaining intersection of $\overline{\mathbf{D P}}_{n}^{\text {HARD }}$ and $\overline{\mathbf{D P}}_{\mathbf{n}}^{\text {SOFT }}$ in terms of operating conditions for cases with constant MR values: (A) 0.05 ; (B) 0.25 ; (C) 0.5 ; (D) 0.75 ; (E) 0.95 .

Likewise, we can also assess the possibility of producing chains with equal or unequal sequence length of ethylenic units. Figure S12 in SI demonstrate that at constant MR values of 0.05 and 0.25 , it is not possible to make balance between $\overline{\text { ESL }}{ }^{H A R D}$ and $\overline{\text { ESL }}$ SOFT and at such conditions it is only possible to produce OBCs with $\overline{\mathrm{ESL}}$ SOFT $<\overline{\mathrm{ESL}}$ HARD (white region is the only possible pattern), which is the reason why these plots were omitted. This is consistent with what one may expect from chain shuttling reaction and the character of Catalyst 2, which makes soft units with high incorporatability of 1-octene comonomer [1, 10, 12-14]. However, further increase of $\mathrm{MR}$ to $0.50,0.75$, and then 0.95 enable the production of OBCs with an inverse behavior of dual Catalyst system where $\overline{\text { ESL }}$ HARD $<\overline{\text { ESL }}$ SOFT (crosshatched red patterns shown in Figure 11). 
(A)
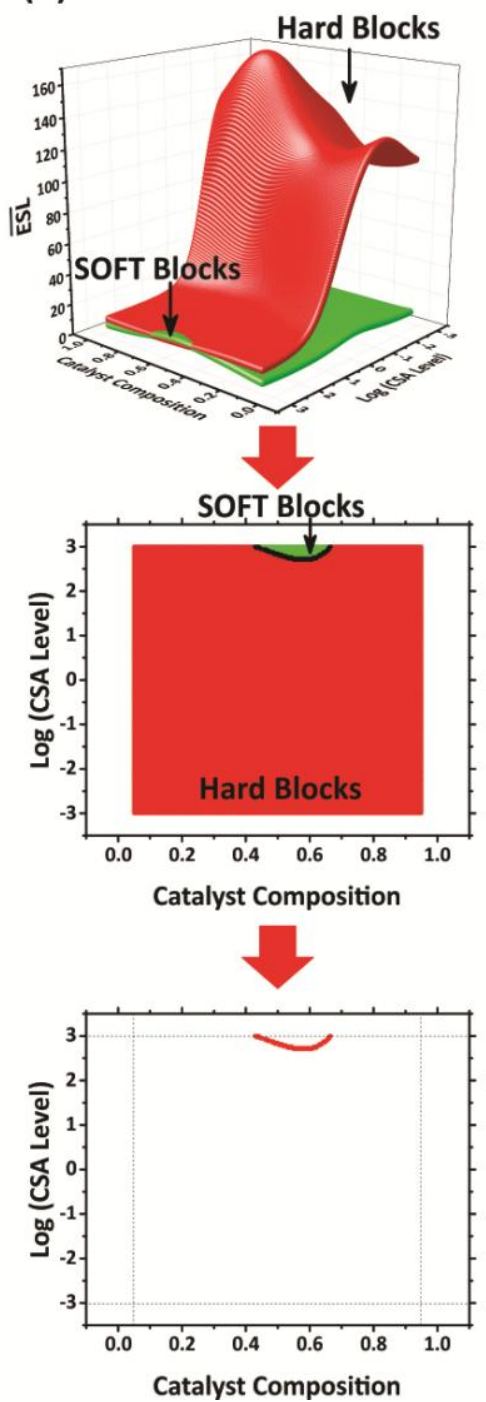

(B)
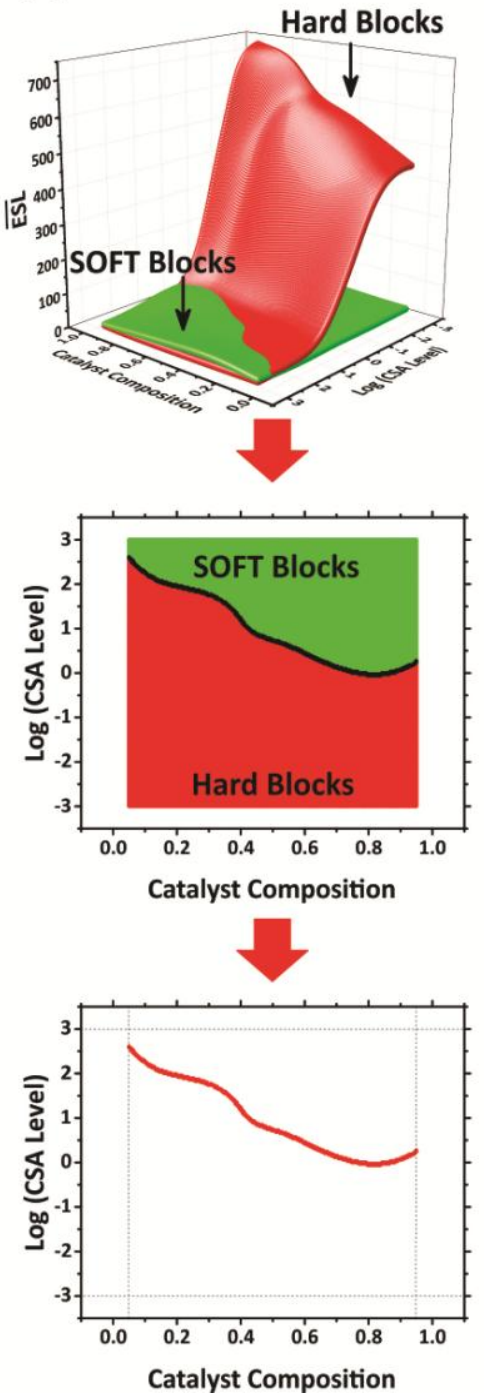

(C)
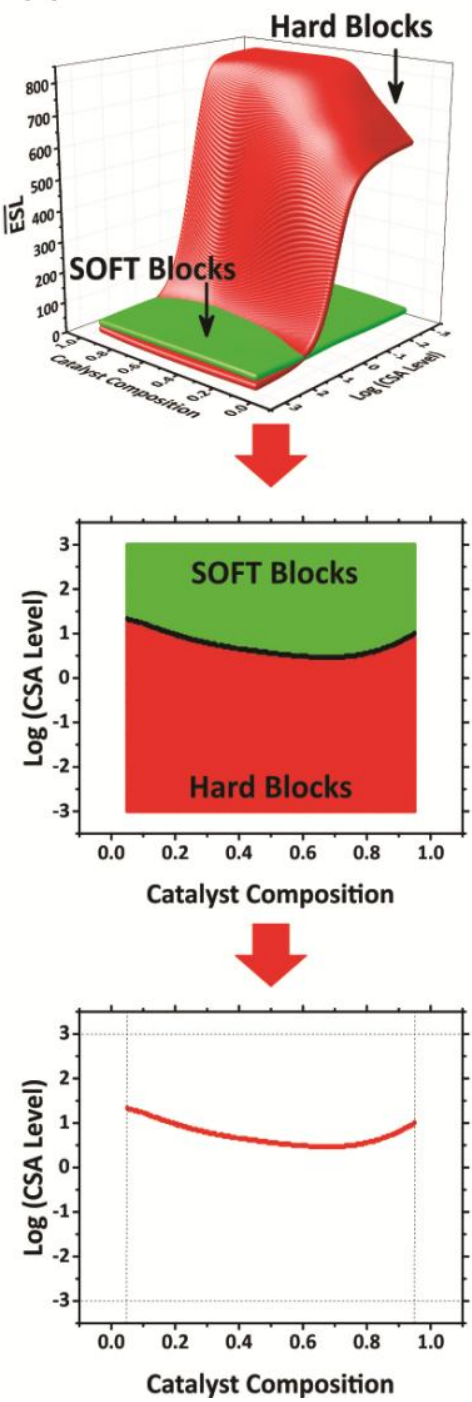

Figure 11. Possibility analysis of obtaining intersection of $\overline{\text { ESL }}$ SOFT and $\overline{\text { ESL }}$ HARD in terms of operating conditions for cases with constant MR values: (A) 0.5 ; (B) 0.75 ; (C) 0.95 .

This rather unexpected result may find its origin in the alteration of active center composition for dual Catalysts systems, as reported by Zhang et al. [29]. The composition of instantaneously formed copolymers originating from Catalysts 1 and 2 deviates from the expected Lewis-Mayo behavior when the chain shuttling rate is higher than the propagation rate. This will basically lead to pseudo-random copolymers.

Catalyst 1 leads in these conditions to higher contents of 1-octene than expected while Catalyst 2 in turn to lower contents of 1-octene than expected. Replacing this in the frame of excess ethylene (e.g. MR 0.75 and 0.95 in the current work) may explain why $\overline{\text { ESL }}$ SOFT can 
become higher than $\overline{\mathrm{ESL}} \mathrm{HARD}$. The trends observed for $\overline{\mathrm{DP}} n$ (hard $>$ soft and hard $<$ soft) may in turn originate from the $\mathrm{CC}$, as predicted by our current model. The amount of the Catalyst combined to its activity may lead to higher $\overline{\mathrm{DP}} n$ from self-shuttling. This is much more pronounced for Catalyst 1 - MR=0.95 - as Catalyst 1 is more active than Catalyst 2, by a factor 10 for ethylene (see Table 1).

The interfacial line is finally the place where $\overline{\mathrm{ESL}}{ }^{\mathrm{SOFT}}=\overline{\mathrm{ESL}}^{\mathrm{HARD}}$. The provided view in Figure 11 indicates that new grades of OBCs can be produced.

A further step forward in producing OBCs would be to control one or two microstructural aspects. The resulting synthesis conditions are illustrated in Figure 12, which address operating conditions needed for production of OBCs with predesigned molecular fingerprints. Five distinguished molecular architectures corresponding to specific operating conditions are typically defined in Scheme 2, in which OBCs with different relationships between microstructural features can be produced. For sake of simplicity, all sample structures in Scheme 2 were drawn with $L P=3$, i.e. 4 blocks, but obviously, LP can be varied (see Fig. 8) to almost any desired level. The last case among possible ones displayed in this Scheme resembles TB-OBC, a random block copolymer, in which the assigned microstructural features $\left(\overline{\mathrm{ESL}}^{\mathrm{HARD}}, \overline{\mathrm{ESL}}^{\mathrm{SOFT}}, \overline{\mathrm{DP}}_{\mathrm{n}}^{\mathrm{HARD}}, \overline{\mathrm{DP}}_{\mathrm{n}}^{\mathrm{SOFT}}\right.$ ) take the same quantity. Regardless of particular properties TB-OBC may show, even imagination of this case before coupling ANN with KMC was impossible. 


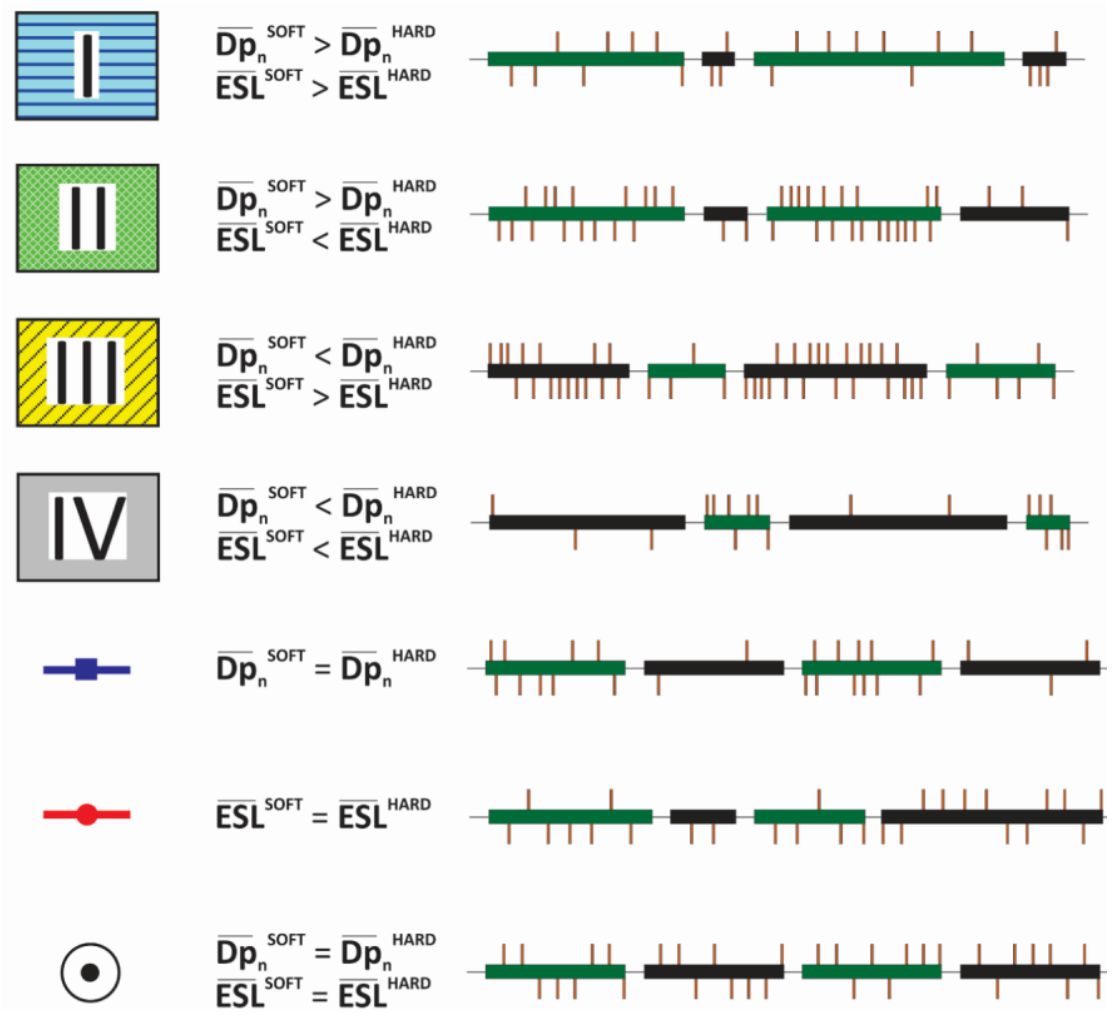

Scheme 2. A snapshot of typical copolymers produced by the combined stochastic model in this work. 
(A)

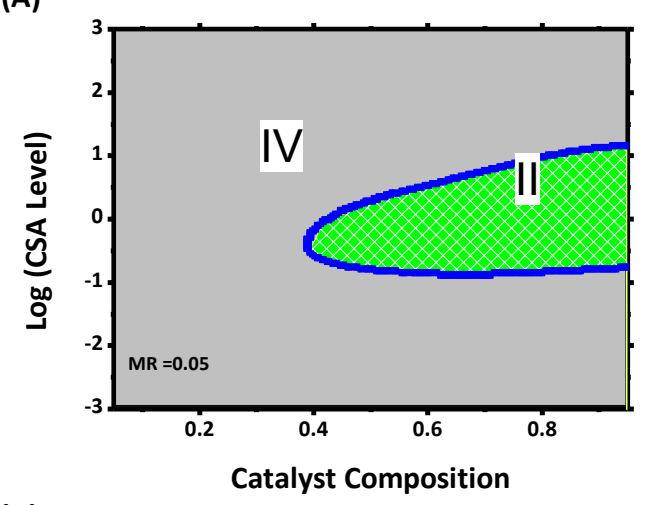

(C)

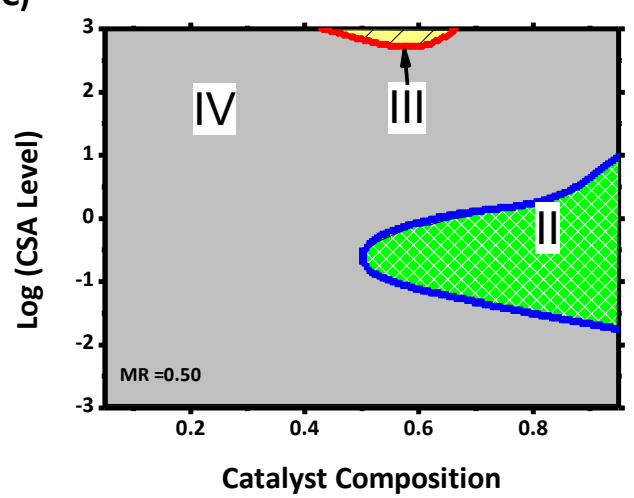

(E)

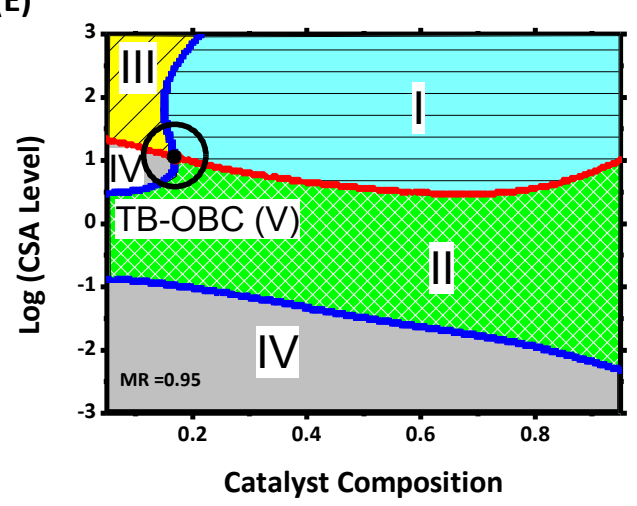

(B)

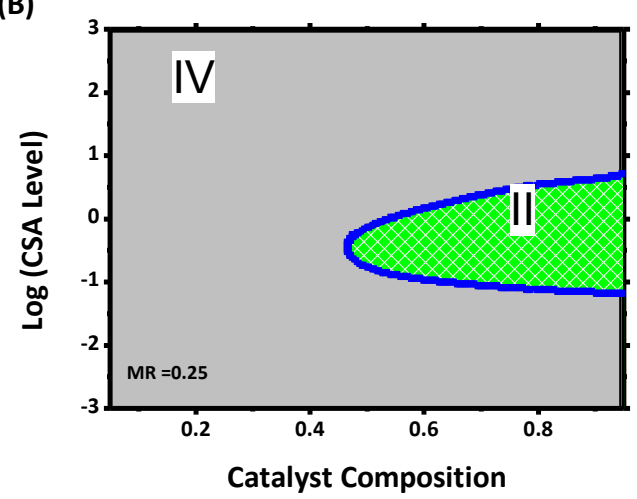

(D)

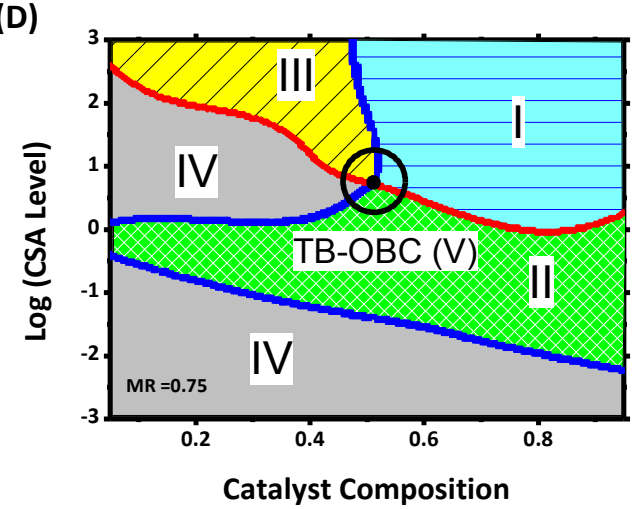

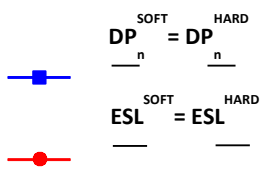

Figure 12. Molecular landscape of $O B C s$ with different $O B C$ regions and possibility of producing TB-OBC at studied range of operating conditions.

Catalysts composition comprising $70 \%$ of Cat 1 and a CSA initial mass of $0.27 \mathrm{~g}$ are typical experimental inputs for the synthesis of $O B C$ reported in the literature [30]. This leads to microstructure (II) independent of the comonomer feed values, as represented by the green areas in Figure 12. Experimentally determined molecular weights of the soft and hard blocks of 
$\mathrm{OBC}$ also follow the trend $\overline{\mathrm{DP}}_{\mathrm{n}}^{\mathrm{HARD}}<\overline{\mathrm{DP}}_{\mathrm{n}}^{\text {SOFT }}$ [31]. Microstructure (iv), where $\overline{\mathrm{ESL}}^{\mathrm{HARD}}>$ $\overline{\mathrm{ESL}}^{\text {SOFT }}$ but $\overline{\mathrm{DP}}_{\mathrm{n}}^{\mathrm{HARD}}>\overline{\mathrm{DP}}_{\mathrm{n}}^{\text {SOFT }}$ are the most common patterns in Figure 12. Shorter soft blocks will probably lead to less elastomeric materials than microstructure (ii). For microstructures (i) and (iii), the ESL of the soft block is higher than that of the hard block, which might lead to particular properties at the macroscopic scale. In plots C, D and E of Figure 12, obtained at MR values of $0.5,0.75$ and 0.95 , the model predicts the formation of OBC with equal ethylene sequence length (red line). The intersection of the red line with the blue line yields a very unique type of $O B C$, we named TB-OBC before, where $\overline{\mathrm{DP}}_{\mathrm{n}}^{\mathrm{HARD}}=\overline{\mathrm{DP}}_{\mathrm{n}}^{\mathrm{SOFT}}$ and $\overline{\mathrm{ESL}}^{\mathrm{HARD}}=\overline{\mathrm{ESL}}^{\mathrm{SOFT}}$. This special grade will probably crystallize in a particular manner, and feature very unique properties at the macroscopic scale. For instance, the operating conditions at which TB-OBC was obtained are, for a feed containing $75 \%$ ethylene ( $M R=0.75$, Figure 16D) are a 50/50 catalyst mixture $(C C=0.5)$, with a CSA charge of 1.42 g (and $\log C S A=0.72$ ). When these values are fed into the ANN model and KMC simulator, a considerable consistency is observed between architectural features (Table 5). Such excellent matching of $\mathrm{KMC}$ and $\mathrm{ANN}$ results would be more appreciated if one considers the significant difference between LV and HV for each response. As a whole, it can be realized that the model developed in this study predicts the formation of new types of $\mathrm{OBC}$, which has never been reported before. The properties of these materials will be studied in a forthcoming paper. 
Table 5. Microstructural characteristics of TB-OBCs predicted by the ANN model at specified condition and the quantities obtained at equivalent condition by KMC simulator.

Operating conditions needed for synthesis of TB-OBCs when MR=0.75 (Figure 16D) and $M R=0.95$ (Figure 16E, given in italic)

\begin{tabular}{lll}
\hline Operating condition & MR=0.75 & MR=0.95 \\
\hline Catalyst Composition & 0.5069 & 0.1690 \\
Log(CSA Level) & $0.7202\left(\right.$ i.e. $\left.0.27 \times 10^{+0.7202} \mathrm{~g}\right)$ & $\begin{array}{l}1.0524\left(\text { i.e. } 0.27 \times 10^{+1.0524} \mathrm{~g}\right) \\
\text { for } 1000 \mathrm{ml} \text { reactor volume }\end{array}$ \\
\hline
\end{tabular}

ANN model and KMC simulator outputs

\begin{tabular}{|c|c|c|c|c|c|}
\hline \multicolumn{6}{|c|}{ Microstructural properties (MR $=0.75 ; M R=0.95$ ) } \\
\hline & $E S L^{\text {Soft }}$ & $E S L^{\text {Hard }}$ & $D P_{n}^{\text {Soft }}$ & $D P_{n}^{\text {Hard }}$ & $L P$ \\
\hline ANN & $22.23 ; 42.02$ & $22.23 ; 42.02$ & $112.02 ; 150.70$ & $112.02 ; 150.70$ & $62.78 ; 54.22$ \\
\hline KMC & $21.15 ; 42.14$ & $22.96 ; 47.34$ & $71.13 ; 142.11$ & $113.34 ; 156.82$ & $59.34 ; 55.03$ \\
\hline LV & 2.34 & 2.19 & 5.13 & 7.12 & 0.00 \\
\hline HV & 48.97 & 825.43 & 38085.6 & 3736.87 & 74.28 \\
\hline Error (\%) & $2.32 ; 0.26$ & $0.09 ; 0.65$ & $0.12 ; 0.02$ & $0.03 ; 0.16$ & $4.63 ; 1.09$ \\
\hline
\end{tabular}

\section{Conclusions}

Molecular landscape of OBC has been patterned in terms of operating conditions including log (CSA level), CC, and MR applying a combinatorial approach. ANN modeling was used to find interrelationship between operating conditions and architectural characteristics of OBCs $\left(\mathrm{LP}, \overline{\mathrm{DP}}_{\mathrm{n}}^{\mathrm{HARD}}, \overline{\mathrm{DP}}_{\mathrm{n}}^{\mathrm{SOFT}}, \overline{\mathrm{ESL}}{ }^{\mathrm{HARD}}\right.$, and $\left.\overline{\mathrm{ESL}}^{\mathrm{SOFT}}\right)$, which was a nearly impossible task by KMC simulator alone.

The following correlations can be found:

1. The number of block links LP is mostly determined by the chain shuttling agent level ( $\log ($ CSA level), Fig. 7 in the main text and Fig. S10 in SI, but also the catalyst composition plays a role. 
a. The influence of the $\log (\mathrm{CSA}$ level) is very obvious for all MR studied as a transition from $L P<1$ for $\log (C S A$ level $)<-2$ to $L P>50$ for $\log (C S A$ level $)>3$. The fact that all subfigures of Fig. 7 look quite similar and that also the catalyst composition only has a rather weak influence suggests that the log(CSA level) is the dominating influence factor for LP, which is logical considering that the OBCsynthesis is a quasi-living polymerization and that the amount of available shuttling agent $(\log ($ CSA level)) and the number of shuttling events taking place are closely related (see Fig. 7F and also Fig. S10 in SI). LP is almost the sole influence factor for LP for very high $\log (\mathrm{CSA}$ level) $(+2$ or +3$)$ or very low $\log (\mathrm{CSA}$ level) -2 or -3 . CC and MR only play a role for intermediate log(CSA levels).

b. The more of the comonomer incorporating catalyst 1 (high CC) is present the less blocks tend to exist for medium and high log(CSA level), while the lowest LP are found for low catalyst composition, i.e. high contents of catalyst 2 producing hard blocks. However, this effect is rather minor, which can be easily visualized by the fact that the change of LP (the dynamic range) for varying log(CSA level) from -3 to 3 is $>100$, while CC-variation only leads to a dynamic range of $1-3$, depending on $\log ($ CSA level).

c. The monomer ratio MR does not play a significant role, which is logical considering the fact that LP only refers to the number of shuttling events per chain and not to the composition of the blocks.

2. The degree of polymerization of soft blocks $\overline{\mathbf{D P}}_{\mathbf{n}}^{\text {SOFT }}$ is to a large extent influenced by all three input parameters (log(CSA level), MR, CC).

a. A low content of octene comonomer in the feed (high MR) leads to a higher average comonomer content and consequently the soft blocks grow faster in comparison to the chain transfer rate, determined by $\log (\mathrm{CSA}$ level). This can be explained by the fact that the ethylene propagation rate is higher than the octene propagation rate for the catalysts used here (and in general for all polyolefin 
catalysts known to the authors) due to steric hindrance. While the different subplots of Fig. 3 look rather similar, one has to check that the color mapping scales differ significantly $-\overline{\mathbf{D P}}_{\mathbf{n}}^{\text {SOFT }}$ increases by approximately factor 6 when increasing MR from 0.05 to 0.95 . This is due to the higher polymerization rate of ethene vs. octene, which allows for a higher number of polymerization steps before the next shuttling event takes place.
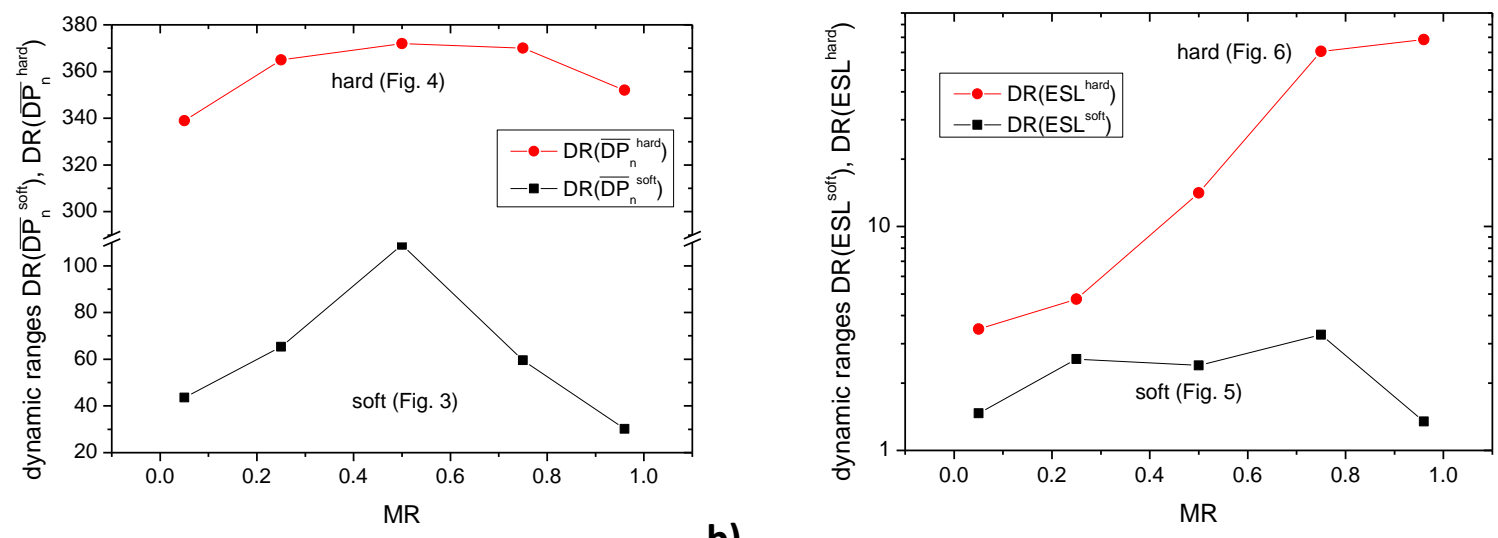

a)

b)

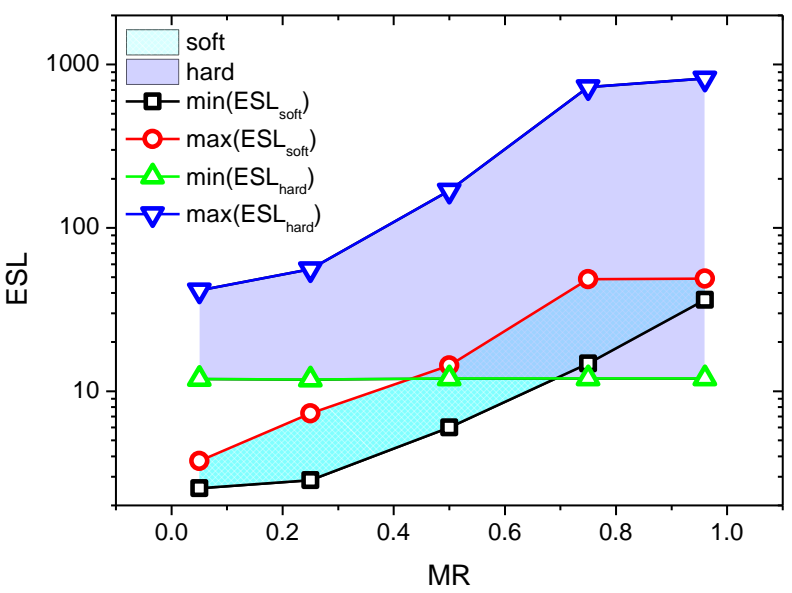

Figure 13. Dynamic range for a) hard and soft segment polymerization degrees $\left(D R\left(\overline{D P}_{n}^{\text {HARD }}\right)\right.$ and $\left.\mathrm{DR}\left(\overline{\mathrm{DP}}_{\mathrm{n}}^{\text {SOFT }}\right)\right)$ and for b) ethylene sequence lengths ( $\overline{\mathrm{ESL}}$ HARD and

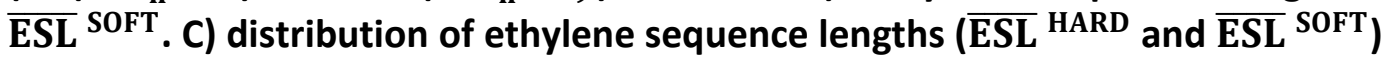

b. The chain shuttling agent level $(\log (\mathrm{CSA}$ level)) determines the average time a growing polymer chain remains attached to an active center and, consequently, it has a direct influence on the degree of polymerization of the soft block $\overline{\mathbf{D P}}_{\mathbf{n}}^{\text {SOFT }}$. 
Fig. 3 shows, however, that the different color maps are quite similar but nevertheless differences in shape of the height lines as well as in the ratio of highest and lowest value are obvious, called dynamic range $\left(D R_{\text {soft }}\right)$. To visualize these differences better, the dynamic range of $\overline{\mathbf{D P}}_{\mathbf{n}}^{\text {SOFT }}$ was calculated from the ratio of the highest and lowest value of $\overline{\mathbf{D P}}_{\mathbf{n}}^{\text {SOFT }}$ and plotted in Figure 13a. For example for $M R=0.05$ (Fig. $3 A$ ) the dynamic range $D R\left(\overline{\mathbf{D P}}_{\mathbf{n}}^{\text {SOFT }}\right)$ of the data is a factor of 109 , while for $M R=0.95$ only a factor of 30 is found. In other words, the $\log$ (CSA level) influences $\overline{\mathbf{D P}}_{\mathbf{n}}^{\text {SOFT }}$ more significantly for intermediate MR. This finding is related to the dependence of LP on $\log ($ CSA level), as obviously a few LP at constant molar mass mean higher $\overline{\mathbf{D P}}_{\mathbf{n}}^{\text {SOFT }}$.

c. The catalyst composition CC influences $\overline{\mathbf{D P}}_{\mathbf{n}}^{\text {SOFT }}$ through changing the probability of self-shuttling, i.e. the more catalyst 1 is present (high CC) the higher is the likeliness that the CSA shuttles the chain to another catalyst 1 . The direct consequence is that the chain continues growing a soft block, thus increasing $\overline{\mathbf{D P}}_{\mathbf{n}}^{\text {SOFT }}$. This influence is clear from Fig. 3 as the more or less straight height lines at an angle for $\log ($ CSA level $)<0.5$

3. $\overline{\mathbf{D P}}_{\mathbf{n}}^{\text {HARD }}$ shows a strong dependence on $\log (\mathrm{CSA}$ level) and much weaker dependencies than $\overline{\mathbf{D P}}_{\mathbf{n}}^{\text {SOFT }}$ on CC and MR.

a. The strong connection between $\overline{\mathbf{D P}}_{\mathbf{n}}^{\text {HARD }}$ and $\log$ (CSA level) (Fig. 4) is obviously due to the shuttling probability, which terminates the hard segment (unless it is shuttled back to catalyst 2). The length of the hard segment $\overline{\mathbf{D P}}_{\mathbf{n}}^{\text {HARD }}$ can be set within a dynamic range $\mathrm{DR}\left(\overline{\mathbf{D P}}_{\mathbf{n}}^{\text {HARD }}\right)$ of approximately a factor of 360 (determined from the ratio of highest to lowest $\overline{\mathbf{D P}}_{\mathbf{n}}^{\text {HARD }}$ ) by adjusting $\log$ (CSA level), see Fig. 13a. 
b. As catalyst 2 incorporates only very little octene, the monomer ratio is more or less not important for $\overline{\mathbf{D P}}_{\mathbf{n}}^{\text {HARD }}$, which can be seen from the almost constant $\overline{\mathbf{D P}}_{\mathbf{n}}^{\text {HARD }}$ at varying MR. This can be easiest assessed from the similarity of Fig. 4ae and the almost identical color scale.

c. $\overline{\mathbf{D P}}_{\mathbf{n}}^{\text {HARD }}$ depends on the catalyst composition $\mathrm{CC}$ in exactly the opposite way as $\overline{\mathbf{D P}}_{\mathbf{n}}^{\text {SOFT }}$ does, due to the same reasons (likeliness of self-shuttling).

4. $\overline{\text { ESL }}$ SOFT shows rather structured plots (Fig. 5), but when checking the dynamic range, it becomes obvious that the only relevant trend is the increase of $\overline{\mathbf{E S L}}$ SOFT with increasing MR (Fig. 13c, showing minimum and maximum values of $\overline{\mathbf{E S L}}$ SOFT as a function of MR). This influence can be understood as the inverse comonomer content of the soft block, which obviously scales with the comonomer in the feed ( $=>M R)$. Only a minor influence of CC (and low $\log (\mathrm{CSA}$ level)) is visible for high MR, which leads to rather high $\overline{\mathbf{E S L}}$ SOFT (increasing from 2-6 to ca. 48 ), see Fig. 13b for the dynamic range plot (DR( $\left.\overline{\mathbf{E S L}}{ }^{\text {SOFT }}\right)$ vs. $\log ($ CSA level)). The reason for this finding is that an increase of MR means a lowering of comonomer content and, consequently, the $\overline{\mathbf{E S L}}{ }^{\text {SOFT }}$ increases as the comonomer content decreases. A significant influence of $\log (\mathrm{CSA}$ level) in general (with the exception of the abovementioned increase for high MR, CC and low $\log (\mathrm{CSA}$ level)) is not present due to the low $\overline{\mathbf{E S L}}$ SOFT, being significantly shorter than the typical block even for high $\log$ (CSA levels).

5. $\overline{\text { ESL }}{ }^{\text {HARD }}$ (Fig. 6) shows a much more significant dependence on the synthesis parameters than $\overline{\mathbf{E S L}}$ SOFT.

a. Due to the significantly higher $\overline{\mathbf{E S L}} \mathbf{H A R D}$, the influence of $\log (\mathrm{CSA}$ level) can be clearly seen, as $\overline{\mathbf{E S L}}$ HARD varies from ca. 10 for $\log ($ CSA level) $=3$ to up to $>800$ for $\log \left(\right.$ CSA level)=-3. The degree of polymerization of the hard block $\overline{\mathbf{D P}}_{\mathbf{n}}^{\text {HARD }}$ is about factor 10-30 higher than $\overline{\mathbf{E S L}}{ }^{\text {HARD }}$. From the rate constants given in Table 
1, it can be obviously understood that transfer to hydrogen rate constant of catalyst 2 is 100 times lower than that of catalyst 1 . This means that catalyst 2 provides higher chance to hard sequences to be grown. As a result, $\overline{\mathbf{D P}}_{\mathbf{n}}^{\text {HARD }}$ becomes more significant, even increases up to 36000 , compared to $\overline{\mathbf{D P}}_{\mathbf{n}}^{\text {SOFT }}$. Moreover, since hard blocks have less comonomers inside and more probability of growth, $\overline{\text { ESL }}$ HARD becomes statistically more sensitive leading to diversity of value of this parameter from 10 to 800 . On the other hand, soft blocks with less probability of propagation and very higher content of comonomer take a more uniform character regarding degree of polymerization. The dynamic range of $\overline{\text { ESL }}$ HARD increases significantly for higher MR (Fig. 13b), which can be understood as the consequence of the "starvation" of catalyst 2 to not get an octene monomer easily for high MR. Hence, shuttling events become more likely instead of chain propagation at high $\log ($ CSA level).

b. The monomer ratio has a strong influence on $\overline{\mathbf{E S L}}{ }^{\text {HARD }}$ at $\operatorname{low} \log (\mathrm{CSA}$ level), while at high $\log (\mathrm{CSA}$ level) the influence is negligible. This can be explained by the finite yet non-negligible tendency of catalyst 2 to incorporate octene. Obviously, the likeliness of octene incorporation is the higher, the higher is the ratio of octene to ethene in the reactor, i.e. the lower is the MR. This tendency cannot be found for high log(CSA level) levels, as the CSA-concentration is so high it becomes the dominating reason to terminate a ethylene sequence.

c. The catalysis composition does not play a significant role for $\overline{\mathbf{E S L}}^{\text {HARD }}$ as long as $\log (\mathrm{CSA}$ level) is high or medium. For low $\log (\mathrm{CSA}$ level) and high MR, high amounts of catalyst 1 (producing soft blocks) lead to an increase of $\overline{\text { ESL }}$ HARD, which can be explained by depletion of octene (accounting for only $5 \%$ of the monomers initially anyway $(\mathrm{MR}=0.95))$. This is shown in Fig. 13c. The almost constant $\min (\overline{\mathbf{E S L}} \mathbf{H A R D})$ is the consequence of the finite tendency of catalyst 2 to incorporate tiny amounts of octene and the high $\log (\mathrm{CSA}$ level), making blocks very short, while at low $\log ($ CSA level) a clear increase of $\overline{\text { ESL }}$ HARD - represented 
by $\max \left(\overline{\mathbf{E S L}}{ }^{\text {HARD }}\right)$ in Fig. 13c can be found. Irrespective of CC, MR, and $\log (\mathrm{CSA}$ level), ethylene is the fast monomer and tends to be consumed very rapidly. Hence, it is expected to have more octene at the later stages of reaction, leading to a significant composition drift. As a consequence of accumulation of octene at later stages of reaction, hard blocks find the chance to obtain more comonomer. This unconditionally at the end of reaction leads to shortening of $\overline{\text { ESL }}$ HARD , as can be seen in Figure 13b from the trend of green triangular symbols. As the simulations were performed assuming a batch-reactor setup, the presence of mostly catalyst 1 will reduce the concentration of octene to a larger extent than at low CC (dominatingly catalyst 2). At low MR (e.g. MR=0.05), the amount of octene in the reactor is so high that the depletion effect is completely irrelevant and, hence, the aforementioned drift does not occur.

The developed model has enabled to establish the relationships between the synthesis parameters and the microstructure of $\mathrm{OBC}$, paving the way for an easier tailoring of $\mathrm{OBC}$. For example, the size of the soft blocks can be tuned by varying the $\mathrm{CC}$ and/or the MR without affecting significantly the length of the hard block. The latter can in turn be adjusted using the quantity of chain shuttling agent in the reactive medium. All synthesis parameters are however interdependent regarding their influence on the microstructure, and applying and examining this hybrid modeling approach, we recognized that the nature of chain shuttling reaction is more intricate than one may imagine. Different molecular landscapes can be established considering the length and ESL of the soft and hard blocks as a function of the synthesis parameters. Having these patterns at hand, one can straightforward choose reaction conditions yielding the desired microstructure patterned with corresponding molecular island. The model predicts finally the formation of completely new $\mathrm{OBC}$, notably TB-OBC with equal length and ESL for both blocks. How these fascinating microstructures self-assemble and behave at macroscopic scale will be discussed in a forthcoming paper.

While the exact ANN results can only be used for the described system, i.e. the system using catalysts with exactly the properties of the ones used for the modeling, the established 
correlations can be used to understand other systems as well to some degree. While the effects will be the same, their exact interplay depends on the properties of the catalyst and the reactor. For example, the aforementioned depletion effect will not be observed for semi-batch or continuously stirred tank reactors, as in these types of reactor the reactant concentration is kept constant.

In summary, it was possible to use relatively few KMC-simulations to create a reliable base for building an ANN network, capable of properly predicting the molecular composition of olefinic block copolymers $(\mathrm{OBC})$. Clear relations were established by the combined KMC-ANN approach with respect to how synthesis parameters influence molecular structure.

While it is not possible to directly make the connections between synthesis parameters and molecular structure due to the complex nature of OBC-polymerization, all KMC-ANN derived correlations could be explained using knowledge on the reaction kinetics. Hence, the obvious disadvantage of an artificial network - it is a black box - could be compensated for by explaining the found correlations by reaction kinetics.

\section{Acknowledgements}

FJS would like to thank the National Science Foundation of China (21574086), Nanshan District Key Lab for Biopolymers and Safety Evaluation (No. KC2014ZDZJ0001A), Shenzhen Sci \& Tech research grant (ZDSYS201507141105130), and Shenzhen City Science and Technology Plan Project (JCYJ20140509172719311) for financial support.

\section{References}

[1] Arriola, D. J.; Carnahan, E. M.; Hustad, P. D.; Kuhlman, R. L.; Wenzel, T. T.; Catalytic production of olefin block copolymers via chain shuttling polymerization, Science 2006, 312, 714-719.

[2] Hustad, P. D.; Frontiers in olefin polymerization: reinventing the world's most common synthetic polymers, Science 2009 , 325, 704-707. 
[3] Alfano, F.; Boone, H. W.; Busico, V.; Cipullo, R.; Stevens, J. C.; Polypropylene "Chain Shuttling" at Enantiomorphous and Enantiopure Catalytic Species: Direct and Quantitative Evidence from Polymer Microstructure, Macromolecules, 2007, 40, 7736-7738.

[4] Xiao, A.; Wang, L.; Liu, Q.; Yu, H.; Wang, J.; Huo, J.; Tan, Q.; Ding, J.; Ding, W.; Amin A. M.; A Novel Linear-Hyperbranched Multiblock Polyethylene Produced from Ethylene Monomer Alone via Chain Walking and Chain Shuttling Polymerization, Macromolecules, 2009, 42, 1834-1837.

[5] Kuhlman, R. L.; Klosin, J.; Tuning Block Compositions of Polyethylene Multi-Block Copolymers by Catalyst Selection, Macromolecules, 2010, 43, 7903-7904.

[6] Wei, J.; Zhang, W.; Wickham, R.; Sita, L. R.; Programmable Modulation of Co-monomer Relative Reactivities for Living Coordination Polymerization through Reversible Chain Transfer between "Tight" and "Loose" Ion Pairs, Angew. Chem. Int. Ed., 2010, 49, 91409144.

[7] Pan, L.; Zhang, K.; Nishiura, M.; Hou, Z.; Chain-Shuttling Polymerization at Two Different Scandium Sites: Regio- and Stereospecific "One-Pot" Block Copolymerization of Styrene, Isoprene, and Butadiene, Angew. Chem. Int. Ed., 2011, 50, 12012-12015.

[8] Descour, C.; Sciarone, T. J. J.; Cavallo, D.; Macko, T.; Kelchtermans, M.; Korobkov, I.; Duchateau, R.; Exploration of the effect of 2,6-( $t-\mathrm{Bu})_{2}-4-\mathrm{Me}-\mathrm{C}_{6} \mathrm{H}_{2} \mathrm{OH}$ (BHT) in chain shuttling polymerization, Polym. Chem., 2013, 4, 4718.

[9] Valente, A. ; Stoclet, G ; Bonnet, F. ; Mortreux, A. ; Visseaux, M. ; Zinck, P. ; Isoprene-styrene chain shuttling copolymerization mediated by a lanthanide half-sandwich complex and a lanthanidocene: straightforward access to a new type of thermoplastic elastomers, Angew. Chem. Int. Ed., 2014, 53, 4638-4641. 
[10] Wenzel, T. T.; Arriola, D. J.; Carnahan, E. M.; Hustad, P. D.; Kuhlman, R. L. Chain shuttling catalysis and olefin block copolymers (OBCs). In: Metal catalysis in olefin polymerization; Topics in Organometallic chemistry 2009, Springer, Berlin, P 65.

[11] Zinck, P.; Unexpected reactivities in chain shuttling copolymerization, Polymer International 2016, 65, 11-15.

[12] Mohammadi, Y.; Ahmadi, M.; Saeb, M. R.; Khorasani, M. M.; Yang, P.; Stadler, F. J.; A Detailed Model on Kinetics and Microstructure Evolution during Copolymerization of Ethylene and 1 - Octene: From Coordinative Chain Transfer to Chain Shuttling Polymerization, Macromolecules 2014, 47, 4778-4789.

[13] Saeb, M. R.; Khorasani, M. M.; Ahmadi, M.; Mohammadi, Y.; Stadler, F. J.; A unified picture of hard-soft segmental development along olefin chain shuttling copolymerization, Polymer 2015, 76, 245-253.

[14] Ahmadi, M.; Saeb, M. R.; Mohammadi, Y.; Khorasani, M. M.; Stadler, F. J.; A Perspective on Modeling and Characterization of Transformations in the Blocky Nature of Ole fin Block Copolymers, Ind. Eng. Chem. Res. 2015, 54, 8867-8873.

[15] Krispel, U.; Schinko, C.; Ullrich, T.; A Survey of Algorithmic Shapes, Remote Sensing 2015, 7, 12763-12792.

[16] Gholamian, M. R.; Fatemi Ghomi, S. M. T.; Ghazanfari, M.; A hybrid intelligent system for multiobjective decision making problems, Computers \& Industrial Engineering 2006, 51, 26-43.

[17] Sjöberg, J; Zhang Q.; Ljung, L.; Benveniste, A.; Deylon, B.; Glorennec, P.-Y.; Hjalmarsson, H.; Juditsky, A.; Nonlinear black-box modeling in system identification: a unified overview, Automatica, 1995, 31, 1691-1724.

[18] Carlile, P. R.; Rebentisch, E. S.; Into the Black Box: The Knowledge Transformation Cycle, Manage. Sci. 2003, 49, 1180-1195. 
[19] Fernandes, F. A. N.; Lona, L. M. F.; Neural network applications in polymerization processes, Braz. J. Chem. Eng. 2005, 22, 401-418.

[20] Zhang, J.; Batch-to-batch optimal control of a batch polymerisation process based on stacked neural network models, Chem. Eng. Sci. 2008, 63, 1273-1281.

[21] Tian, Y.; Zhang, J.; Morris, J.; Modeling and Optimal Control of a Batch Polymerization Reactor Using a Hybrid Stacked Recurrent Neural Network Model, Ind. Eng. Chem. Res. 2001, 40, 4525-4535.

[22] Zhang, J.; Morris, A. J.; Martin, E. B.; Kiparissides, C.; Prediction of Polymer Quality in Batch Polymerization Reactors Using Robust Neural Networks, Chem. Eng. J. 1998, 69, 135-143.

[23] D’Anjou, A.; Torrealdea, F. J.; Leiza J. R.; Asua, J. M.; Arzamendi, G.; Model Reduction in Emulsion Polymerization Using Hybrid First-Principles/Artificial Neural Network Models, Macromol. Theory Simul. 2003, 12, 42-56.

[24] Zhang, J.; A Reliable Neural Network Model Based Optimal Control Strategy for a Batch Polymerization Reactor, Ind. Eng. Chem. Res. 2004, 43, 1030-1038.

[25] Ng, C. W.; Hussain, M. A.; Hybrid neural network-prior knowledge model in temperature control of a semi-batch polymerization process, Chem. Eng. Process. 2004, 43, 559-570.

[26] Gonzaga, J. C. B.; Meleiro, L. A. C.; Kiang, C.; Maciel Filho, R.; ANN-based soft-sensor for real-time process monitoring and control of an industrial polymerization process, Comput. Chem. Eng. 2009, 33, 43-49.

[27] Minari, R. J.; Stegmayer, G. S.; Gugliotta, L. M.; Chiotti, O. A.; Vega, J. R.; Industrial SBR Process: Computer Simulation Study for Online Estimation of Steady-State Variables Using Neural Networks, Macromol. React. Eng. 2007, 1, 405-412.

[28] Bates, F. S.; Hillmyer, M. A.; Lodge, T. P.; Bates, C. M.; Delaney, K. T.; Fredrickson, G. H.; Multiblock polymers: panacea or Pandora's box?, Science 2012, 336, 434-440. 
[29] Zhang, M.; Carnahan, E. M.; Karjala, T. W.; Jain, P.; Theoretical Analysis of the Copolymer Composition Equation in Chain Shuttling Copolymerization, Macromolecules 2009, 42, 8013-8016.

[30] Arriola, D.J.; Carnahan, E.M.; Cheung Y.W.; Devore D.D.; Graf D.D.; Hustad D.D.; Kuhlman R.L.; Li Pi Shan C.; Poon B.C.; Roof G.R.; Stevens J.C.; Stirn P.J.; Wenzel T.; Catalyst composition comprising shuttling agent for ethylene multi-block copolymer formation, US Patent Publication WO2005090427 A2, 2005.

[31] Li, S.; Register, R. A.; Weinhold, J. D.; Landes, B. G.; Melt and Solid-State Structures of Polydisperse Polyolefin Multiblock Copolymers, Macromolecules 2012, 45 (14), 57735781. 


\section{Graphical Abstract}

Unspoken aspects of chain shuttling reactions: Patterning the molecular landscape of olefin multi-block copolymers

Mohammad Reza Saeb ${ }^{1}$, Yousef Mohammadi ${ }^{2 *}$, Tayebeh Sadat Kermaniyan ${ }^{3}$, Philippe Zinck ${ }^{4}$, Florian J. Stadler ${ }^{*}$

${ }^{1}$ Department of Resin and Additives, Institute for Color Science and Technology, P.O. Box 16765-654, Tehran, Iran.

${ }^{2}$ Petrochemical Research and Technology Company (NPC-rt), National Petrochemical Company (NPC), P.O. Box 14358-84711, Tehran, Iran.

${ }^{3}$ Department of Polymer Engineering and Color Technology, Amirkabir University of Technology, Tehran, Iran.

${ }^{4}$ University of Lille, ENSCL, UCCS, UMR CNRS 8181, Bât C7, Cité Scientifique, 59652 Villeneuve d'Ascq Cédex, France.

${ }^{5}$ College of Materials Science and Engineering, Shenzhen Key Laboratory of Polymer Science and Technology, Guangdong Research Center for Interfacial Engineering of Functional Materials, Nanshan District Key Lab for Biopolymers and Safety Evaluation, Shenzhen University, Shenzhen 518060, China.
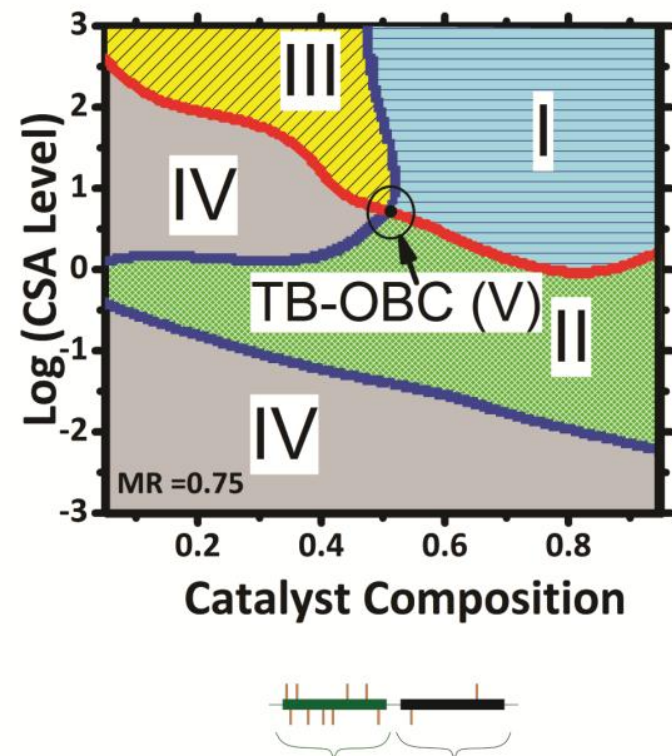

SOFT Blocks HARD Blocks

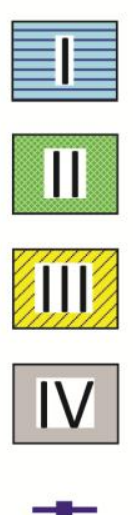

$\overline{\mathrm{Dp}}_{\mathrm{n}}{ }^{\mathrm{OOF}}>\overline{\mathrm{Dp}}_{\mathrm{n}}^{\text {HARD }}$

$\mathrm{ESL}^{\text {Sofr }}>\mathrm{ESL}^{\text {HaRD }}$

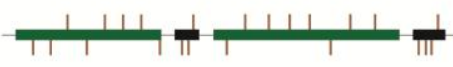

$\overline{\mathrm{Dp}}^{\text {}}{ }^{\mathrm{OOFT}}>\overline{\mathrm{Dp}}_{\mathrm{n}}{ }^{\text {HARD }}$

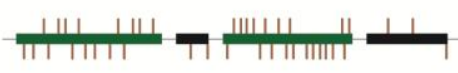

$\mathrm{ESS}^{\mathrm{SOFT}}<\mathrm{ESL}^{\mathrm{HARO}}$

$\overline{\mathrm{Dp}}_{\mathrm{n}}{ }^{\text {OOF }}<\overline{\mathrm{Dp}}_{\mathrm{n}}{ }^{\text {HARD }}$

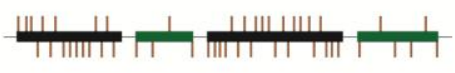

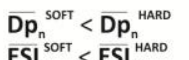

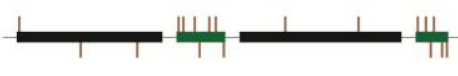

$\overline{D p}_{n}{ }^{\text {SOFT }}=\overline{\mathrm{Dp}}_{\mathrm{n}}{ }^{\text {HARD }}$
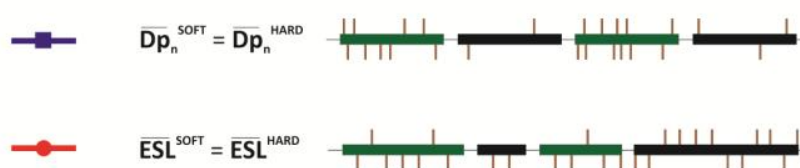

$\overline{E S L}^{\text {SOFT }}=\overline{E S L}^{\text {HARD }}$

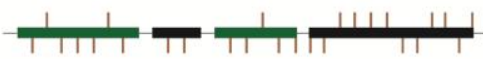

(-)

$\overline{\mathrm{Dp}}_{\mathrm{n}}^{\text {SOFT }}=\overline{\mathrm{Dp}}_{\mathrm{n}}^{\text {HARD }}$

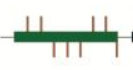

